\title{
THEMIS observations of double-onset substorms and their association with IMF variations
}

\author{
C.-C. Cheng ${ }^{1}$, C. T. Russell ${ }^{2}$, V. Angelopoulos ${ }^{2}$, I. R. Mann $^{3}$, K.-H. Glassmeier ${ }^{4,5}$, and W. Baumjohann ${ }^{6}$ \\ ${ }^{1}$ Department of Electronic Engineering, National Formosa University, Hu-Wei 63201, Taiwan \\ ${ }^{2}$ Department of Earth and Space Sciences and Institute of Geophysics and Planetary Physics, University of California, \\ Los Angeles, CA 90095, USA \\ ${ }^{3}$ Department of Physics, University of Alberta, Edmonton, Alberta T6G 2J1, Canada \\ ${ }^{4}$ Technical University of Braunschweig, Germany \\ ${ }^{5}$ Max-Planck Institute for Solar System Research, Katlenburg-Lindau, Germany \\ ${ }^{6}$ Space Research Institute, Austrian Academy of Sciences, Graz 8042, Austria
}

Received: 13 June 2010 - Revised: 24 February 2011 - Accepted: 27 February 2011 - Published: 28 March 2011

\begin{abstract}
On 16 July 2008, two pairs of consecutive bursts of Pi2 pulsations were recorded simultaneously across the THEMIS ground-based observatory system. Wavelet transformation reveals that for each high-latitude pair, the dominant frequency of the first burst is higher than that of the second. But at low latitudes, the dominant frequency does not change. It is suggested that both pairs result from fast magnetospheric cavity waves with the second burst also containing shear Alfvén waves. INTERMAGNET magnetograms at auroral latitudes showed magnetic variations affected by two recurrent electrojets for each pair. The ground-based magnetometers and those at geostationary orbit sensed magnetic perturbations consistent with the formation of the substorm current wedge. Four consecutive enhancements of energetic electron and ion fluxes detected by the THEMIS probes in the dayside magnetosphere appeared in the later afternoon and then in the early afternoon. The horizontal magnetic variation vectors had vortex patterns similar to those induced by the upward and downward field-aligned currents during substorm times. The hodogram at mid- $L$ stations had a polarization pattern similar to the one induced by the substorm current wedge for each Pi2 burst. The mapping of ground Pi2 onset timing to the interplanetary magnetic field (IMF) observations shows that they appear under two cycles of north-to-south and then north variation. CLUSTER 4 in the south lobe observed wave-like magnetic fluctuations, probably driven by near-Earth reconnection, similar to those on the ground. These two observations are consistent with the link of double-onset substorms to magnetotail reconnection externally triggered by IMF variations.
\end{abstract}

Correspondence to: C.-C. Cheng (cccheng@nfu.edu.tw)
Keywords. Magnetospheric physics (MHD waves and instabilities; Solar wind-magnetosphere interactions; Storms and substorms)

\section{Introduction}

As reviewed by Baker et al. (1996), substorm disturbances in the near-Earth magnetotail commonly include energetic particle injections, the formation of a current wedge, auroral breakups, high latitude magnetic bays and $\mathrm{Pi} 2$ pulsations. The latter disturbances are the impulsive and damped geomagnetic oscillations with a frequency band of $6-25 \mathrm{mHz}$ (corresponding to a period 40-150 s) (see Baumjohann and Glassmeier, 1984; Yumoto, 1986; Olson, 1999, and references of therein). In addition, Pi2 bursts can occur successively in a sequence (e.g. Saito, 1969; Clauer and McPherron, 1974) in association with the variations of the interplanetary magnetic field (IMF) (e.g. Cheng et al., 2005, 2009a, b). Determining how these successive bursts are triggered and their relation to the onset of auroral activations is still an important and unresolved topic.

Over the past four decades, two prevailing and competing scenarios, the near-Earth neutral line (NENL) model (e.g. Hones, 1984) and the current disruption (CD) model (e.g. Lui, 2000), have been proposed as explanations for the development and occurrence sequence of hydromagnetic disturbances in the near-Earth magnetotail during substorms. Both models are famously in disagreement on the cause of substorm onsets. The NENL model suggests the neutral line formation to be the substorm cause and the CD model attributes the near-Earth current disruption instead. Importantly neither of these models directly address why substorm-like activations can have a sequence of multiple onsets. More recently,

Published by Copernicus Publications on behalf of the European Geosciences Union. 


\section{THEMIS \& INTERMAGNET}

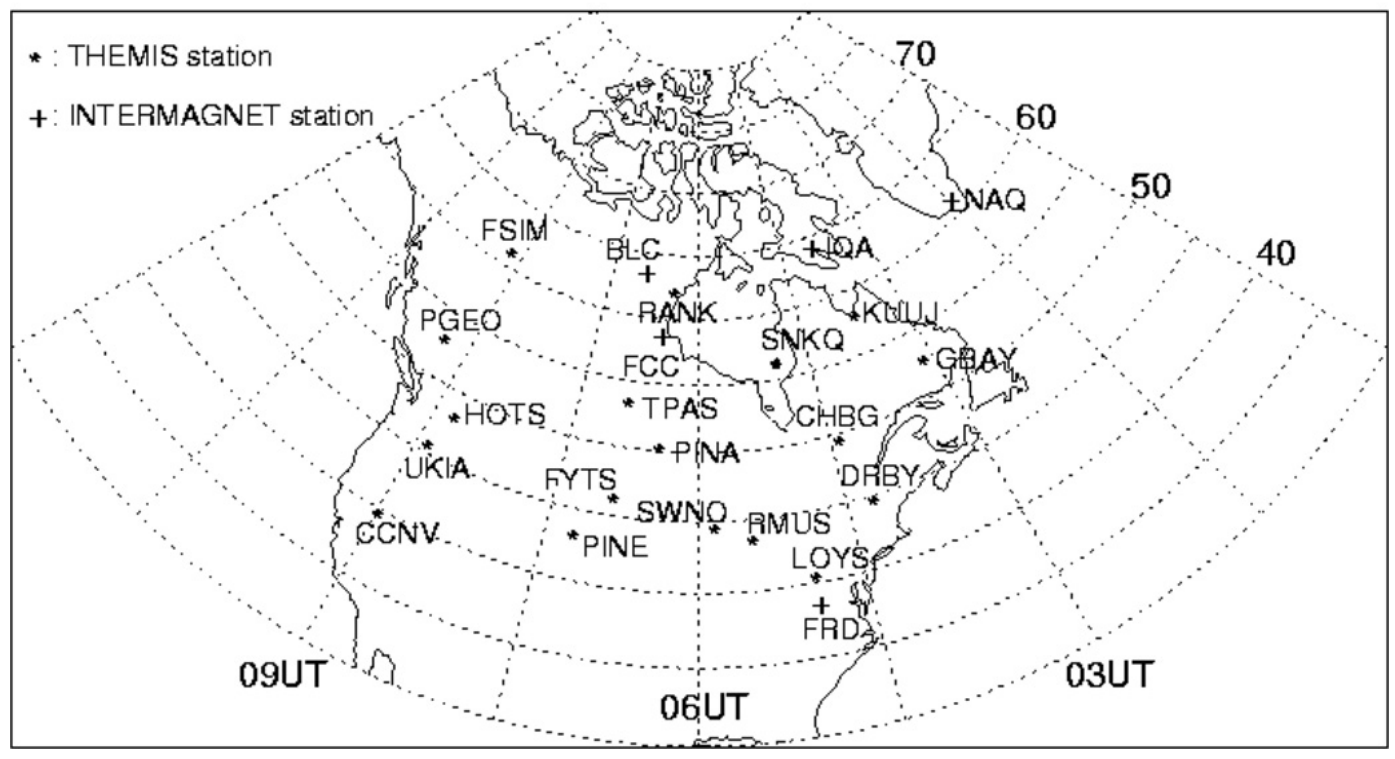

Fig. 1. The locations of the ground-based observatories for the THEMIS mission and four INTERMAGNET observatories for this study. The sign + denotes the INTERMAGNET observatory with a three-letter abbreviation and the sign $*$ the THEMIS station with a four-letter one.

to explain these double-onset observations (e.g. Mishin et al., 2001), Russell (2000) discussed aspects of the near-Earth neutral point that were implicit in the original neutral point model by Russell and McPherron (1973). The neutral point model is similar to the NENL model but emphasizes the necessary localized nature of the onset of time-varying reconnection at the near-Earth neutral point, especially as manifested in flow bursts. Russell (2000) refined the near-Earth neutral point model by noting that the distant and near-Earth neutral points interact. The interplay between the two neutral points in the magnetotail creates two onsets, one when reconnection at the near-Earth neutral point begins on closed field lines within the plasma sheet, and the second when the nearEarth neutral point reaches the open flux of the tail lobes, magnetically linking to the outer neutral point and releasing the plasmoid (Russell, 1974). In this model, the timing of the second substorm onset is controlled by the distant neutral point that in turn is controlled by the northward turning of the IMF. This scenario in which the IMF variation goes from north-to-south and north again, has been shown to be consistent with the systematic observations of the IMF and consecutive Pi2 bursts by Cheng et al. (2002a, b). However, these studies did not have the more comprehensive wellcoordinated measurements in space and on the ground that are available now. These observations can provide further verification of the wave modes of consecutive Pi2 bursts and their connection with the impulsive onsets in the near-Earth magnetotail in response to the IMF variations during doubleonset substorms.
On the other hand, earlier studies (Caan et al., 1975; McPherron et al., 1986; Sergeev et al., 1986) reported that substorm onsets are associated with northward turning of the IMF. Subsequent work (e.g. Lyons, 1996; Hsu and McPherron, 2002, and references of therein) suggested that northward IMF turning could play the role of a trigger in substorm onsets. However, Morley and Freeman (2007) argue that northward IMF turning is not necessary for substorm onset. We note that these studies test whether every IMF northward turning leads to substorm onset. Their conclusions come from single-satellite observations with an assumed solar wind propagation time delay.

Unlike previous works, the present study focuses on two double-onset substorms occurring under two IMF variation cycles of north-to-south and then north. For each substorm, the first onset does not have to occur with external triggering. To lessen the uncertainty of using a single-satellite observation, we adopted the approach used by Cheng et al. (2009a, b) that the incident IMF observed by the satellite in the upstream region is mimicked as propagating to $\sim 1 \mathrm{AU}$ just in front of bow shock (corresponding to $\mathrm{XGSM} \sim 17.0 R_{\mathrm{E}}$ ) with minimum variance analysis (e.g. Weimer et al., 2003) and then compared to the in-situ observation just in front of the Earth's magnetopause. Hence, this approach can ascertain the association of double-onset substorms with IMF variations.

Recently, the instrumentation deployed by the THEMIS mission (Time History of Events and Macroscale Interactions during Substorms; see Angelopoulos, 2008), includes auroral imagers, ground magnetometers and space-borne 

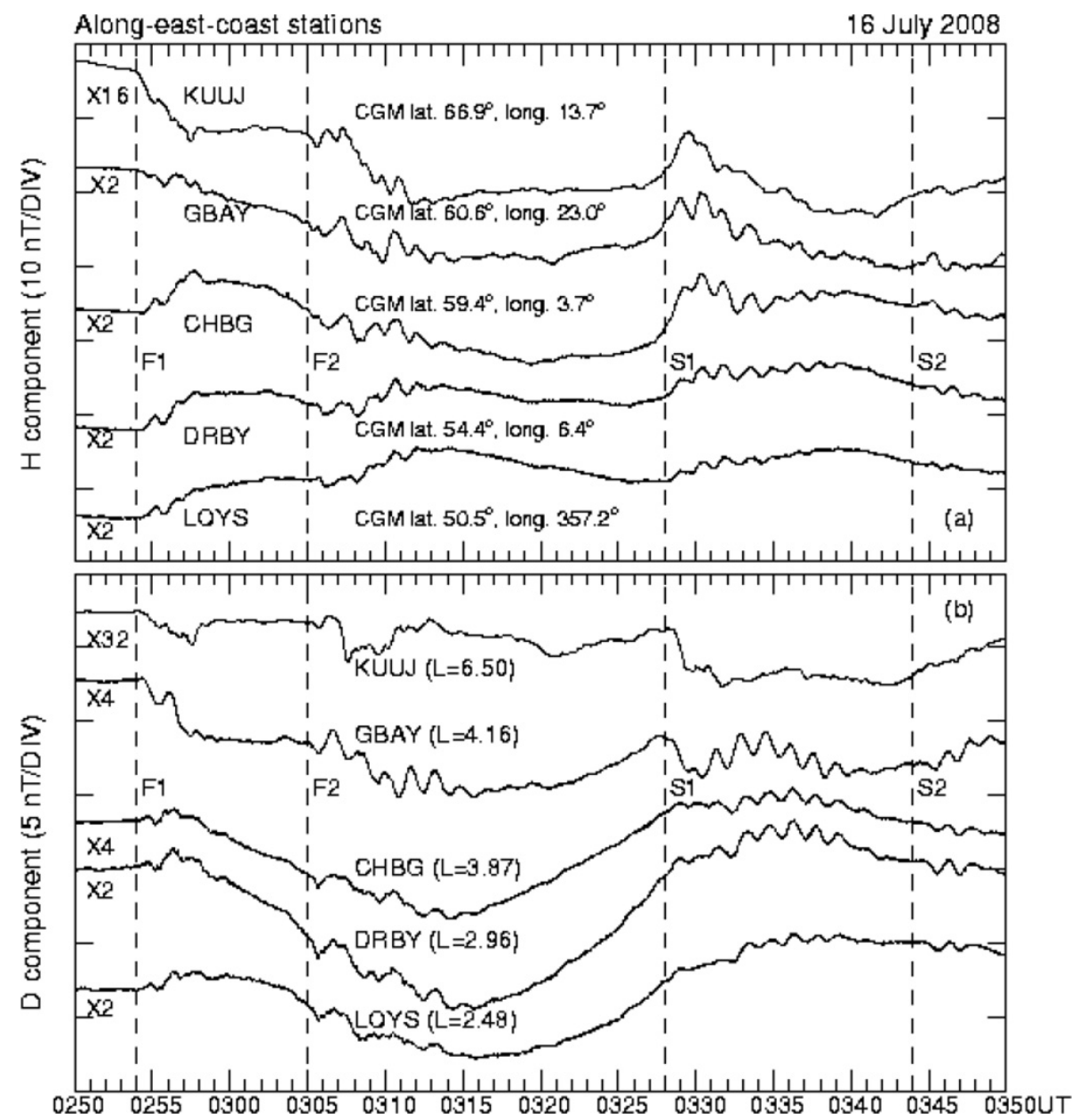

Fig. 2. The H- and D-components at the ground stations along the East Coast of North America from 02:50 UT to 03:50 UT on 16 July 2008. The vertical dashed lines denote ground Pi2 onsets. The letter F denotes the first substorm and the letter S denotes the second one. For each substorm, two consecutive Pi2 onsets are marked with a serial number, respectively.

measurements. Owing to the Earth's annual orbit around the Sun, the THEMIS probes are not always in the magnetotail. Thus their apogees can be in the dayside, flank and tail regions. During the dayside acquisition phase, the THEMIS probes can detect the incident solar wind conditions just in front of the dayside magnetopause, while the nightside magnetospheric responses can be monitored using auroral and/or magnetic measurements on the ground as well as other conjunction probes. From 02:50 UT to 03:50 UT on 16 July 2008, two pairs of consecutive Pi2 bursts occurred simultaneously at the THEMIS ground magnetometers. Meanwhile, four THEMIS probes were in the dayside except the THEMIS-C probe in low orbit moving into the nightside. THEMIS-A, THEMIS-D and THEMIS-E probes were orbiting in the afternoon sector and the THEMIS-B probe, provided the IMF measurements at $\mathrm{XGSM} \sim 25.0 R_{\mathrm{E}}$, in a distance upstream of magnetopause that left little uncertainty as to arrival time. The geostationary satellites GOES 11 and GOES 12 were moving in the dusk sector and in the pre- midnight sector, respectively. The CLUSTER 4 probe was located in the south lobe of the nightside magnetosphere. These observations together with complementary upstream and ground measurements provided us an opportunity to study the link between consecutive Pi2 bursts, the impulsive onsets in the near-Earth magnetotail and the IMF variations during double-onset substorms.

\section{Data presentation}

\subsection{Ground magnetic measurements}

Figure 1 shows the locations of THEMIS ground observatories marked with an asterisk and four INTERMAGNET stations marked with a plus sign. Figure 2 shows the time series of the $\mathrm{H}$ - and $\mathrm{D}$-components from high to low latitude stations along the East Coast of North America from 02:50 UT to $03: 50$ UT on 16 July 2008 . One can see that there were four Pi2 bursts successively occurring at 02:54 UT, 03:05 UT, 

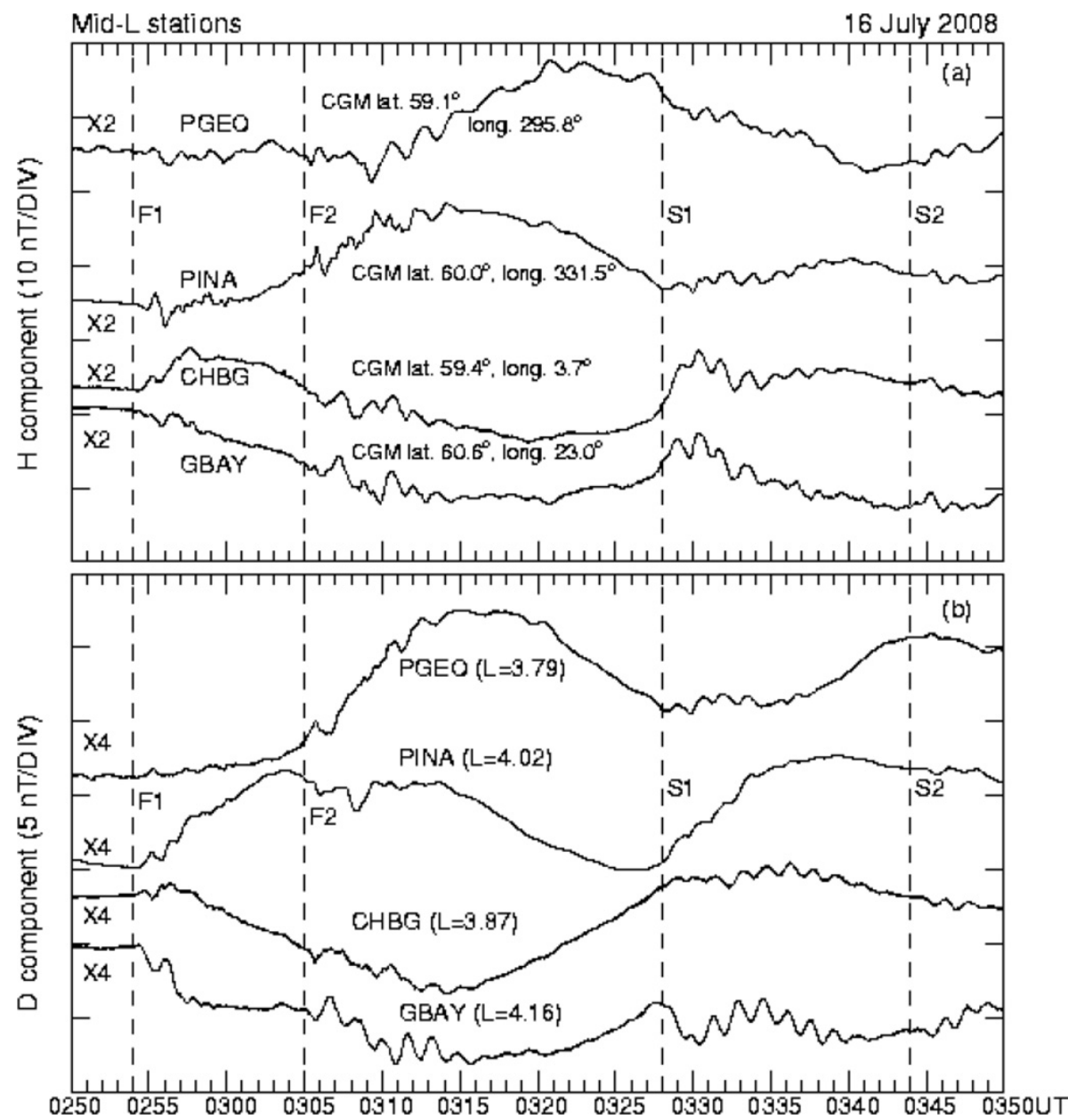

Fig. 3. In the same format as Fig. 2, except for the mid- $L$ stations near $L=4$.

03:28 UT and 03:44 UT, respectively. By comparing to the INTERMAGNET magnetograms shown in the later section during the time of interest, one can see that two Pi2 pairs occurred in two distinct periods of aurora-related electrojet activations corresponding to two consecutive substorms, each having double onsets. Hence, the letter F denotes the first substorm and the letter $\mathrm{S}$ the second substorm in this study. Moreover, for each substorm, two consecutive onsets are marked with a serial number. The vertical dashed line denotes each onset. In this study, the time separation between Pi2s is $\sim 11$ min for the first substorm and $\sim 16$ min for the second substorm, respectively. The separation between the F2 and $\mathrm{S} 1$ bursts is $\sim 23 \mathrm{~min}$. The detailed description of THEMIS ground observatories and instrumentations is given by Russell et al. (2008) and accessible at the website (http://themis.ssl.berkeley.edu/instrument_gmags.shtml).

One can find from Fig. 2a that in contrast to the positive bay-like fluctuations at CHBG (corrected geomagnetic (CGM) latitude 59.4 ${ }^{\circ}$, DRBY (CGM lat. 54.4 ${ }^{\circ}$ ) and LOYS (CGM lat. 50.5 ), the H-component at KUUJ (CGM lat. $66.9^{\circ}$ ) has a clearly negative bay-like perturbation and that at GBAY (CGM lat. 60.6 ) has a weak one at the F1 and $\mathrm{S} 1$ onsets. For the F2 onset, the H-component at KUUJ first fluctuated without a clear magnitude change and then had negative bay-like perturbations after 03:07 UT. In contrast, GBAY and CHBG sensed the weak negative bay-like perturbations after the F2 onset. The H-component at DRBY and LOYS initially had weak negative bay-like perturbations and positive bay-like ones $3 \mathrm{~min}$ after the F2 onset. As for the S2 onset, the magnitude of the H-component at three lowlatitude stations tended to slightly decline. At KUUJ and GBAY, the H-component became enhanced prior to the S2 onset and then had weak negative bay-like perturbations after 03:46 UT. Hence for each substorm, the magnitude of the Hcomponent becomes more enhanced at lower latitude stations at the first onset than at the second onset. In addition, the $\mathrm{H}$-component at higher latitude stations has a more clearly negative bay than that at lower latitude stations. As for the D-component in Fig. 2b, the Pi2 amplitude at KUUJ and GBAY became larger than that at other low-latitude stations. Moreover, the D-component at KUUJ and GBAY has a more clearly negative bay than that at other low-latitude stations. 
Wavelet transformation of $\mathrm{H}$ comp. at THEMIS stations along east coast
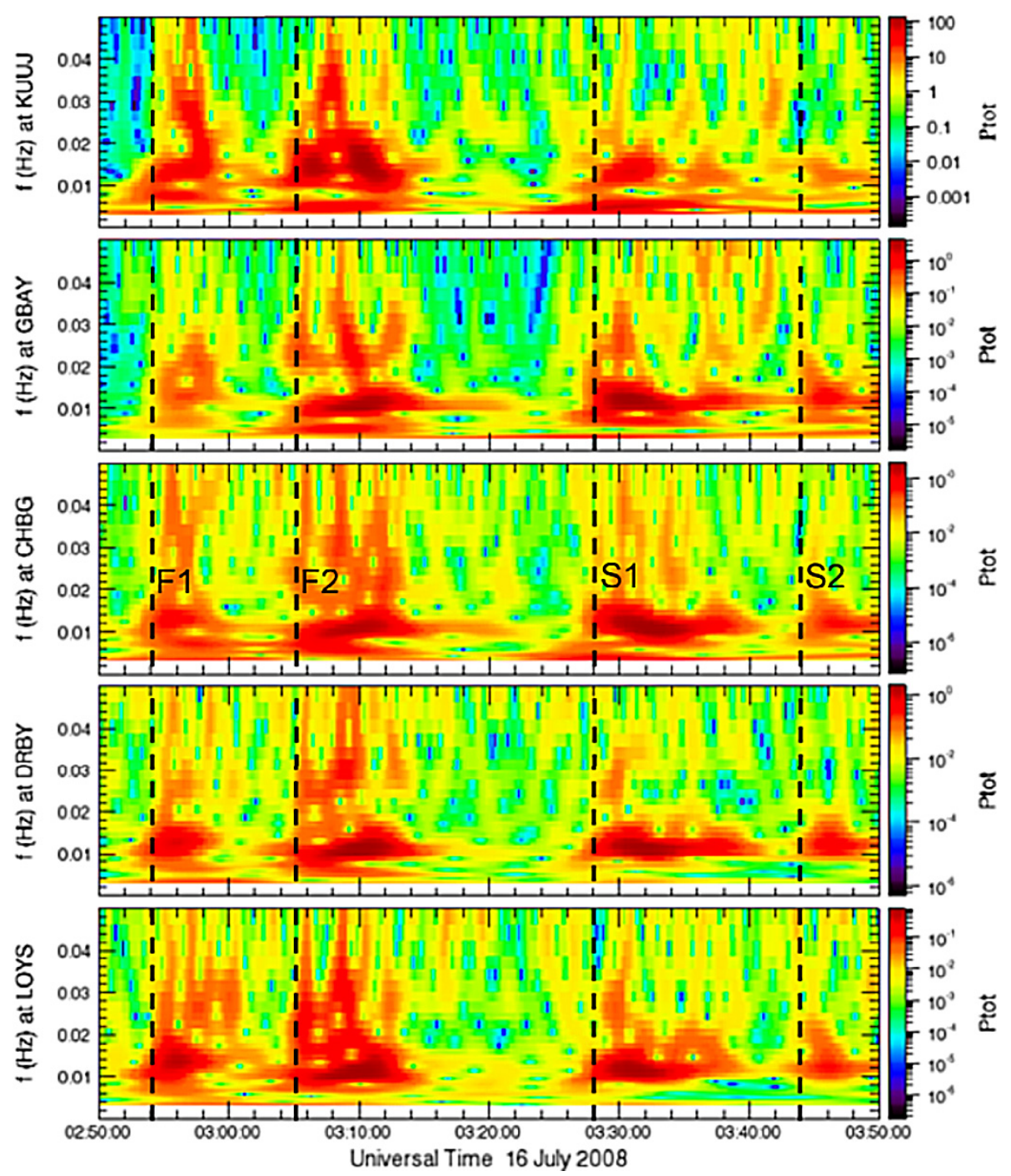

Fig. 4. The wavelet transformation of the H-component at the THEMIS ground stations along the East Coast of North America from 02:50 UT to 03:50 UT on 16 July 2008. The vertical dashed lines denote ground Pi2 onsets.

These magnetic disturbances resemble the ones affected by the downward field-aligned current in the right wing of the substorm current wedge (e.g. Sakurai and McPherron, 1983). Thus, these ground observations signify the occurrence of the downward field-aligned current linking to the westward electrojet in the auroral ionosphere as those during substorms.

To verify the above inference, we also examined the ground magnetometer data at the mid- $L$ stations. In the same format as Fig. 2, Fig. 3 shows the time series of the H- and D-components at the mid- $L$ stations near $L=4$. Note that the PINA data is from the CARISMA array, the instrumentation details of which can be found in Mann et al. (2008) and on the website (http://www.carisma.ca). As for the F1 onset, CHBG recorded a strong positive magnetic bay in the $\mathrm{H}$-component different from three other stations. The $\mathrm{H}-$ component at PINA initially fluctuated and then had a weak positive magnetic bay. PGEO sensed the wave-like perturbations without any clear magnitude change. GBAY had wavelike perturbations accompanied by a decline in background magnitude after the F1 onset. As for the F2 onset, both GBAY and CHBG have negative bays in contrast to PGEO (CGM lat. $59.1^{\circ}$ ) and PINA (CGM lat. $60.0^{\circ}$ ) with positive ones. After the S1 onset, the H-component at CHBG and GBAY has a more clearly wave perturbation than PGEO and PINA. But as for the $\mathrm{S} 2$ onset, four ground stations appear to simultaneously sense a very weak but clear fluctuation. It is noteworthy in Fig. 3b that the D-component at GBAY has a negative bay-like perturbation but a positive one at PINA, located westward in a longitudinal separation from GBAY, for each substorm. This indicates that the upward field-aligned 
Wavelet transformation of $D$ comp. at THEMIS stations along east coast

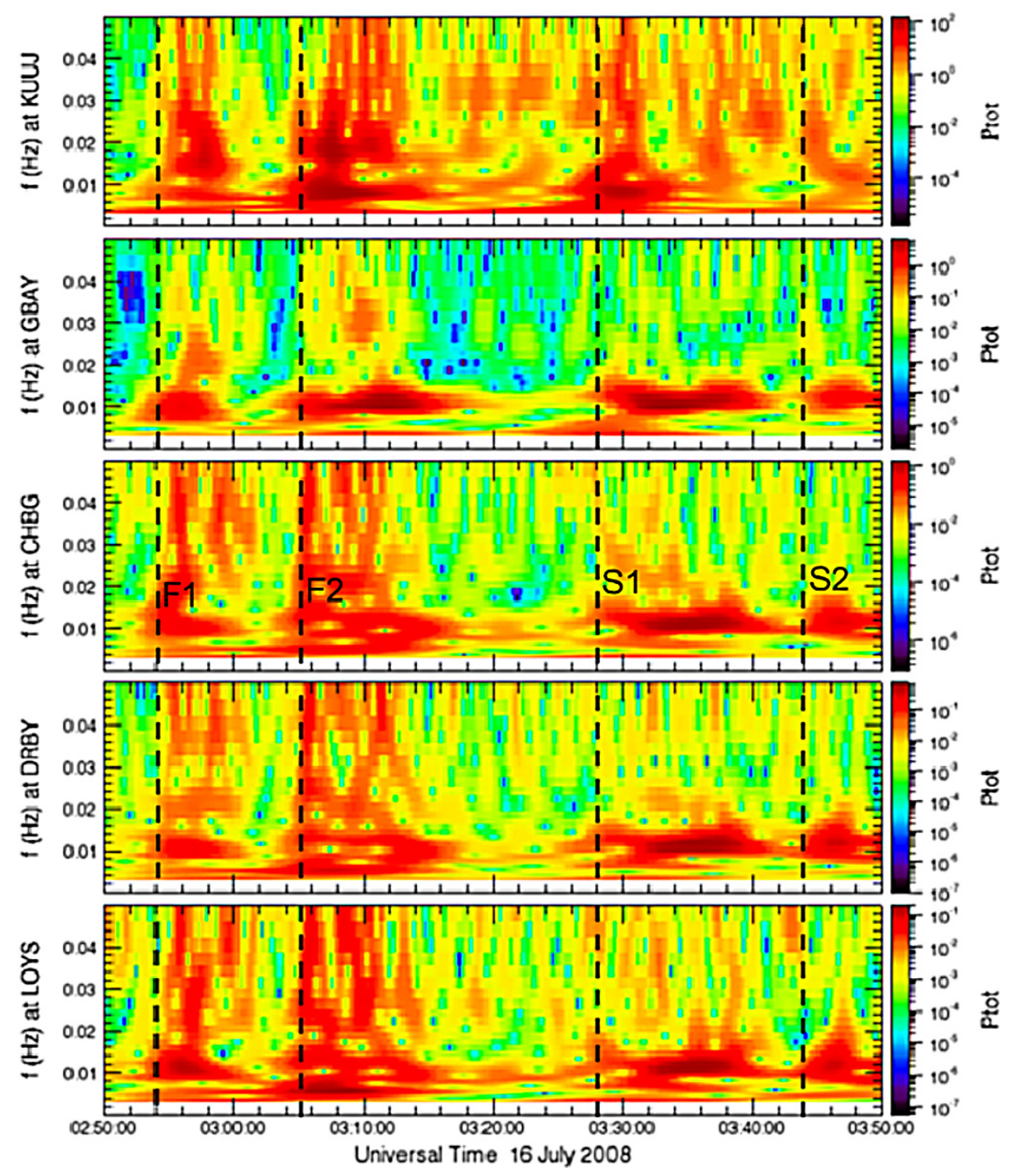

Fig. 5. In the same format as Fig. 4, except for the D-component.

current is located near PINA during the formation of the substorm current wedge (SCW) confirming the SCW formation occurring at each $\mathrm{Pi} 2$ onset.

\subsection{Spectral analysis}

Since the spectral analysis of Pi2 bursts at the stations in the same meridian can provide the information on their source mechanisms and location, we turn to perform the wavelet transformation of consecutive Pi2s at the THEMIS observatories along the East Coast of North America during the time of interest in this subsection. Figure 4 shows the wavelet transformation of the H-component from 02:50 UT to 03:50 UT on 16 July 2008. There are four clear power enhancements for each station with the same frequency band around $0.01 \mathrm{~Hz}$ (corresponding to the period $100 \mathrm{~s}$ ) in Fig. 4.
In contrast, the power enhancements at KUUJ $(L=6.50)$ extend over a broader frequency than those at other stations. On the other hand, Fig. 2 shows that for each Pi2 burst along the East Coast the waveforms at KUUJ $(L=6.50)$ seem out of phase with those at the stations at lower latitudes. But from GBAY ( $L=4.16)$ down to LOYS $(L=2.48)$, their waveforms are almost in the same phase. The detailed Pi2 phase relationship can be clearly discerned from the waveform comparison after removing the ambient magnetic field. These Pi2 phase relationships can also be seen in the waveform comparison shown in Figs. 6 and 15. It is well accepted (e.g. Olson, 1999, and references of therein) that the $\mathrm{H}$-component of the ground magnetometer can sense the compressional component of fast mode waves propagating across the ambient magnetic field to the Earth without any 
phase change. Moreover, fast mode waves can be trapped in the magnetospheric cavity and result in a cavity resonance with $180^{\circ}$ of phase change in the H-component from high to low latitudes across the plasmapause footpoint (see Lin et al., 1991; Cheng et al., 1998). The dominant frequency of the H-component at five stations along the East Coast meridian is the same as that of the compressional component for a fast magnetospheric cavity mode. The dominant frequency is close to the second harmonic frequency of plasmaspheric cavity mode reported by Takahashi et al. (2003). The discussion of why our interpretation of the wave mode is different from theirs is left to a later section. Moreover, one can find from Fig. 4 that the distribution of power enhancements at DRBY $(L=2.96)$ and LOYS $(L=2.48)$ is more centered at the frequency $\sim 0.01 \mathrm{~Hz}$ than that at KUUJ $(L=6.50)$, GBAY $(L=4.16)$ and CHBG $(L=3.87)$ spreading to less than $0.01 \mathrm{~Hz}$. This implies that Pi2s at DRBY $(L=2.96)$ and LOYS $(L=2.48)$ can be dominantly fast magnetospheric cavity resonances but those at KUUJ $(L=6.50)$, GBAY ( $L=4.16)$ and CHBG $(L=3.87)$ may be a mixture of fast magnetospheric cavity resonance and shear Alfvén modes as Itonaga and Yumoto (1998) suggested. In the same format as Fig. 4, Fig. 5 shows the D-component. One can see from Fig. 5 that for the first Pi2 pair, the power enhancements of D-component at KUUJ seem to have a broader and higher frequency band than those at low latitudes. Except for GBAY having the dominant frequency $\sim 0.01 \mathrm{~Hz}$, the power enhancements of D-component of four other stations at the second onset seem to expand to lower frequency band in addition to the dominant frequency band at the first onset. But for the last pair, the power enhancements of D-component seem to be unchanged at the frequency $\sim 0.01 \mathrm{~Hz}$ except for KUUJ ( $L=6.50$ ) having a broader and higher frequency band at the first onset than that at the second onset. The comparison of Fig. 5 with Fig. 4 shows that the pulsation power enhancements after the F1 and S1 onsets appear to be in a broad band with a centered frequency of $\sim 0.01 \mathrm{~Hz}$ but the dominant frequency band is less than $0.01 \mathrm{~Hz}$ for the F2 and S2 onsets.

To further verify the findings from the wavelet transformation, we have compared the waveforms of the two Pi2 bursts for each substorm. After removing the ambient magnetic field, each pair of Pi2 bursts are compared over a time interval of $12 \mathrm{~min}$. Fig. 6 shows the waveforms of H- and D-components for each pair. For each substorm, the waveform of the second burst is shifted right to let its first peak align with that of the first burst. The blue trace denotes the first burst and the red trace the second burst, respectively. The vertical line denotes the adjacent amplitude peak of $\mathrm{H}$ and D-components and the horizontal line the distinct wave period of each burst, respectively. For the first substorm in Fig. 6a, the waveform of the H-component at KUUJ looks like broad band perturbations different from those at other stations having monochromatic-like ones. At KUUJ, the distinct wave period of the F1 burst is $\sim 60 \mathrm{~s}$ (correspond-

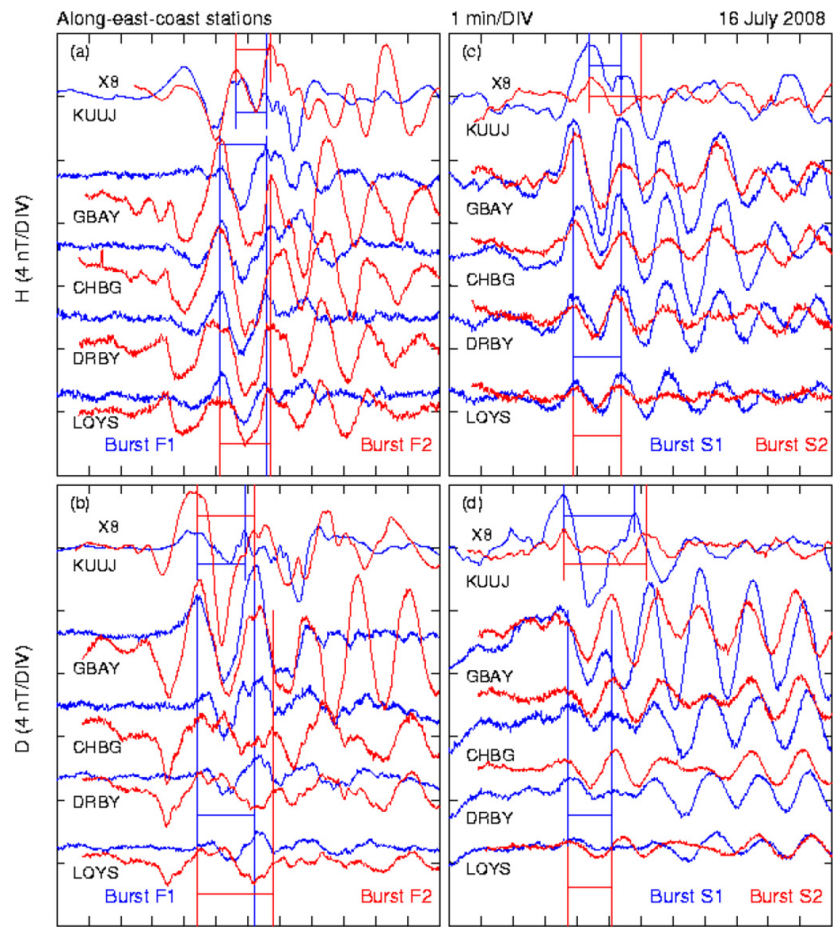

Fig. 6. (a) The H-component at the THEMIS ground stations along the East Coast of North America for the F1 and F2 bursts on 16 July 2008. The blue trace denotes the F1 burst and the red trace the F2 burst. The blue horizontal line denotes the distinct wave period of the F1 burst and the red horizontal line the F2 burst. (b) In the same format as (a), except for the D-component. (c) In the same format as (a), except for the S1 and S2 bursts. (d) In the same format as (c), except for the D-component.

ing to $16.6 \mathrm{mHz}$ ) less than that of the $\mathrm{F} 2$ burst $\sim 66 \mathrm{~s}$ (corresponding to $15.1 \mathrm{mHz}$ ). For four other stations, the distinct wave period of the $\mathrm{F} 1$ burst is $\sim 84 \mathrm{~s}$ (corresponding to $11.9 \mathrm{mHz}$ ) close to that of the F2 burst $\sim 90 \mathrm{~s}$ (corresponding to $11.1 \mathrm{mHz}$ ). As for the D-component of the first substorm shown in Fig. 6b, the waveform of the F1 burst at KUUJ becomes more perturbed with high frequency than that of the F2 burst. For the F1 burst, the waveform at GBAY looks monochromatic in contrast to those at four other stations bearing irregular ones. As for the F2 burst, KUUJ and GBAY sensed clear waveforms but three other stations still recorded irregular ones. At KUUJ, the distinct wave period of the F1 burst is $\sim 90 \mathrm{~s}$ (corresponding to $11.1 \mathrm{mHz}$ ) less than that of the F2 burst $\sim 108 \mathrm{~s}$ (corresponding to $9.2 \mathrm{mHz}$ ). For four other stations, the distinct wave period of the F1 burst is $\sim 108 \mathrm{~s}$ (corresponding to $9.2 \mathrm{mHz}$ ) less than that of the F2 burst $\sim 144 \mathrm{~s}$ (corresponding to $6.9 \mathrm{mHz}$ ). One can see from Fig. $6 \mathrm{c}$ and $\mathrm{d}$ that the H- and D-components of the $\mathrm{S} 1$ and S2 bursts have Pc-like waveforms except for KUUJ sensing damped perturbations. For the $\mathrm{H}$-component at KUUJ, the distinct wave period of the $\mathrm{S} 1$ burst is $\sim 60 \mathrm{~s}$ (corresponding to $16.6 \mathrm{mHz}$ ) less than that of the $\mathrm{S} 2$ burst 
$\sim 96 \mathrm{~s}$ (corresponding to $10.4 \mathrm{mHz}$ ). For four other stations, the distinct wave period of the $\mathrm{S} 1$ burst is $\sim 74 \mathrm{~s}$ (corresponding to $13.5 \mathrm{mHz}$ ) the same as that of the $\mathrm{S} 2$ burst. As for the D-component at KUUJ, the distinct wave period of the S1 burst is $\sim 132 \mathrm{~s}$ (corresponding to $7.5 \mathrm{mHz}$ ) less than that of the S2 burst $\sim 156 \mathrm{~s}$ (corresponding to $6.4 \mathrm{mHz}$ ). For four other stations, the distinct wave period of the S1 burst is $\sim 84 \mathrm{~s}$ (corresponding to $11.9 \mathrm{mHz}$ ) the same as that of the $\mathrm{S} 2$ burst. As a result, the waveform comparison of $\mathrm{H}-$ and D-components at each burst is consistent with the findings from the wavelet transformation. This shows that the dominant frequency band of ground Pi2s at the first onset becomes higher than that at the second onset for each substorm. It is well known that nightside $\mathrm{Pi} 2$ waves excited by the impulsive source in the magnetotail can propagate across the magnetospheric cavity in fast modes and along the geomagnetic field lines to the ground in shear Alfvén modes (e.g. Cheng et al., 2004, 2009a, b). Hence, the dominant frequency of ground $\mathrm{Pi} 2 \mathrm{~s}$ depends on their source location and traveling path that is determined by the cavity size, the length of the field lines and the plasma density in the magnetosphere. In other words, their source location at the first onset can map to the Earth at lower latitudes than that at the second onset.

According to Russell (2000), reconnection at the nearEarth neutral point in the closed field in the plasma sheet leads to the first onset. Later, the second onset results from reconnection at the near-Earth neutral point progressing from the closed field lines of the plasmas sheet onto the open field of the lobes due to the unchanged pressure between the closed field lines in the plasma sheet and those in the tail lobes. It is possible that the source for the first onset could be closer to the Earth than that for the second one. Fast waves and shear Alfvén waves can result from magnetic reconnection at the near-Earth neutral point. Since the plasmapause is not a fixed boundary, not all fast waves from the tail are trapped in the plasmaspheric cavity. For the first onset, fast waves bouncing between the near-Earth neutral point and the Earth can be transmitted through the plasmapause at a node to excite a magnetospheric cavity resonance mode during their duration time. But for the second onset, shear Alfvén waves bouncing back along the open field lines in between the auroral ionosphere and the tail lobes can drive field line oscillations over the duration of their source. Reconnection can cause the field line configuration and magnetospheric cavity size to change more drastically than the plasma density. Owing to the unchanged plasma density on the path of the Pi2 waves, their frequency can be dominantly affected by the cavity size and the field line length. The frequency of the cavity-mode Pi2 is higher than that of the shear Alfvén waves as the cavity size is less than the field line length. This may be the reason why the dominant frequency of ground Pi2s at the first onset can be higher than that at the second onset.

\subsection{INTERMAGNET magnetograms}

Since the THEMIS keograms were not well suited for substorm characterization during the time of interest, we turn to the INTERMAGNET magnetograms at auroral latitudes to determine whether aurora-related electrojet activations occurred at each ground $\mathrm{Pi} 2$ onset in this subsection. Figure 7 shows the $\mathrm{X}$ - and Z-components at BLC (CGM lat. $73.4^{\circ}$, long. $328.9^{\circ}$ ), IQA (CGM lat. $72.1^{\circ}$, long. $14.7^{\circ}$ ), FCC (CGM lat. $68.4^{\circ}$, long. $333.4^{\circ}$ ), NAQ (CGM lat. 65.6 ${ }^{\circ}$, long. $42.6^{\circ}$ ), and FRD (CGM lat. $48.5^{\circ}$, long. $358.5^{\circ}$ ) from 02:30 UT to 04:00 UT, respectively. Since RANK (CGM lat. $72.3^{\circ}$, long. $335.9^{\circ}$ ) is close to BLC, the X- and Zcomponents at RANK are also shown in Fig. 7 for comparison. The locations of five INTERMAGNET observatories are shown on Fig. 1. For detailed instrumentations of the INTERMAGNET observatories we refer to the website (http://www.intermagnet.org). The dashed vertical lines denote ground Pi2 onsets. One can see from Fig. 7a that the $\mathrm{X}$-component at FCC started to decrease and at FRD, to increase at $\sim 02: 54 \mathrm{UT}$. Later, the X-component at NAQ first decreased prior to those at BLC, RANK and IQA sharply dropped at $\sim 03: 28 \mathrm{UT}$. But FCC and FRD observed enhanced $X$ at the same time. At $\sim 03: 44 \mathrm{UT}$, the X-component appeared to clearly decrease at BLC, RANK and IQA in contrast to other stations. Figure $7 \mathrm{~b}$ shows that the Z-component at NAQ decreased prior to those at FCC and FRD declined at $\sim 02: 54$ UT. The decrease in $Z$ at FCC further intensified at $\sim 02: 57$ UT. Subsequently, the Z-component at FCC decreased prior to the declines of NAQ and FRD at $\sim 03: 04$ UT. In contrast, at that time, BLC and RANK sensed a strong $Z$ enhancement. At $\sim 03: 25 \mathrm{UT}$, the Z-component at FCC increased and those at NAQ and FRD decreased. These features further intensified at $\sim 03: 28 \mathrm{UT}$ while BLC observed an enhanced $Z$. Finally, the Z-component at BLC declined before 03:44 UT, followed by slight enhancements at IQA and FCC. These INTERMAGNET observations suggest that the westward electrojet first activated over FCC at the F1 onset as evidenced by the decrease in the $\mathrm{H}$-component at 02:54 UT. A subsequent decrease in the H-component observed at FCC revealed a further intensification of westward electrojet occurred at $\sim 03: 04$ UT. There was an additional activation over BLC and RANK occurring further poleward at $\sim$ 03:08 UT. While a second positive H-component excursion and related D-component signatures at lower latitude at $\sim 03: 28$ UT appear to be consistent with a second substorm onset, one may argue that it could be a simple intensification of the westward electrojet portion of the original wedge segment, the onset of which was $\sim 02: 54 \mathrm{UT}$. The $X$ and $Y$ polarities and magnitudes at FRD (Y-component not shown in this study) for the $\sim 02: 54$ UT onset and the $\sim 03: 28$ UT intensification look similar to those in Fig. 2, indicating they take place in roughly the same longitude range. Further inspection of the NAQ magnetogram suggests the westward electrojet much further east intensified at $\sim 03: 28 \mathrm{UT}$, one would 


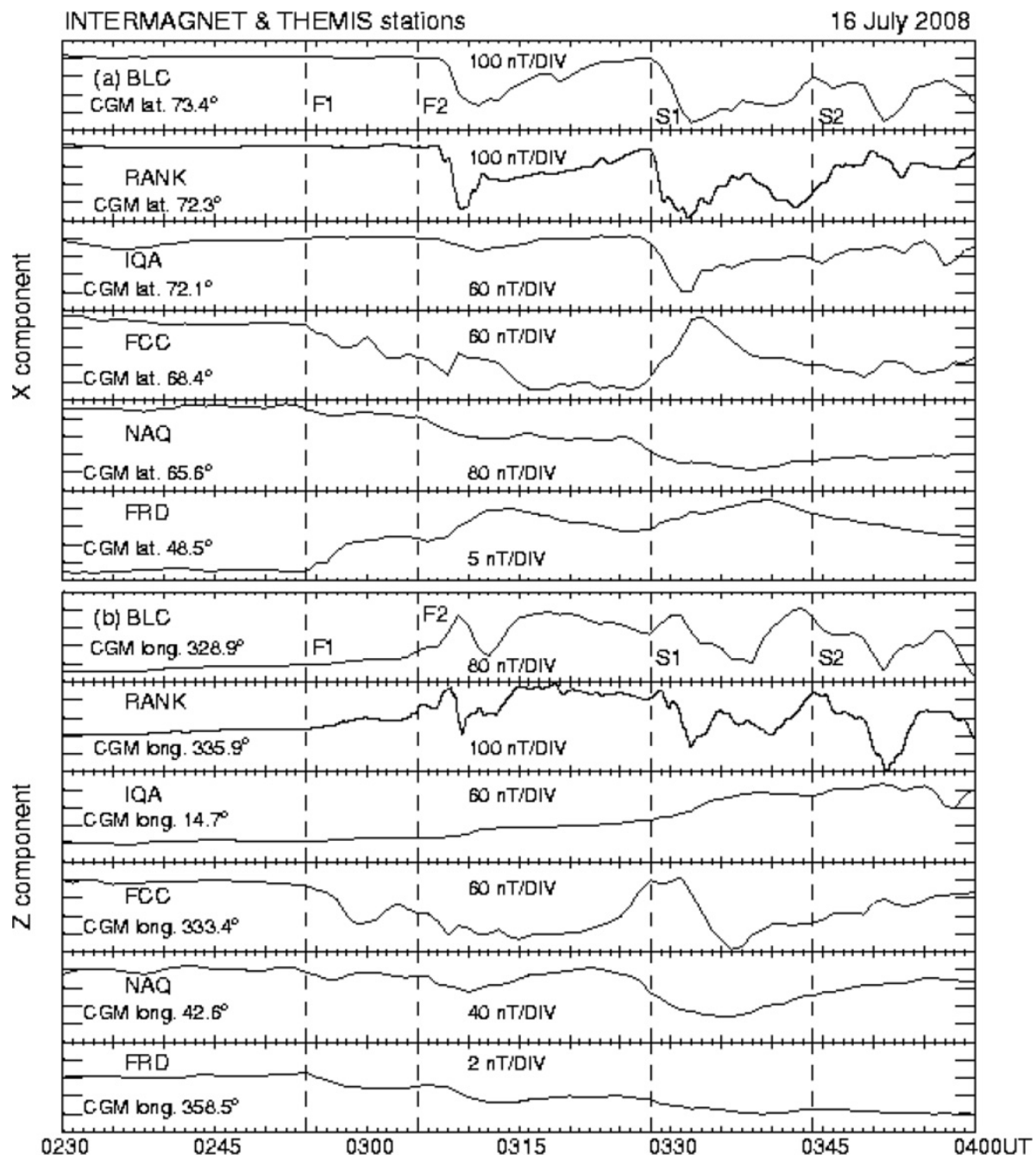

Fig. 7. (a) The X-component at the available INTERMAGNET stations and RANK from 02:30 UT to 04:00 UT on 16 July 2008. The vertical dashed lines denote ground Pi2 onsets. (b) In the same format as (a), except for the Z-component.

expect that the wedge westward electrojet occurred at the latitude of the original onset and intensified some distance poleward afterwards. Moreover at the S1 onset, the X-component at BLC and RANK decreased. The Z-component at BLC increased and that at RANK initially increased and then decreased. This suggests that the westward electrojet is close to BLC and expands equatorward toward RANK. As for the S2 onset, the X-component at BLC decreased and that at RANK increased. The Z-component at BLC decreased prior to the S2 onset and that at RANK decreased with a larger magnitude post the $\mathrm{S} 2$ onset. This indicates that the westward electrojet is at higher latitude than BLC and expands equatorward toward RANK.

Without comparison to two discrete pairs of Pi2s simultaneously occurring at lower latitudes, one may have a view that these four onsets are simply a continuation of the substorm that started at $\sim 02: 54 \mathrm{UT}$, and the activity occurs at the poleward edge of the (already) expanded auroral bulge.
Thus, four aurora-related westward electrojets occurred in two distinct periods in which the first one is related to the first substorm and the latter the second substorm, identified from variations of the $\mathrm{AL}$ index shown in the later section. To justify our argument of two double-onset substorms, we note the observations on the website (http://aurora.iar.nagoya-u. ac.jp/southpole/) that auroral breakups with expansion movements (not shown in this study) occurred over the South Pole at each Pi2 onset. According to Rostoker (2002), the characteristic feature of auroral breakup after substorm onset is a brightening accompanied with both poleward and equatorward movements. In contrast, there is a tendency in Fig. 7 for the aurora-related westward electrojet at the first onset to appear at lower latitude than that at the second onset for each activation period. This feature is consistent with the findings by Cheng et al. (2005) with the meridian scanning photometer data. Namely, the above tendency in the INTERMAGNET magnetograms justifies the inference on the source sites 


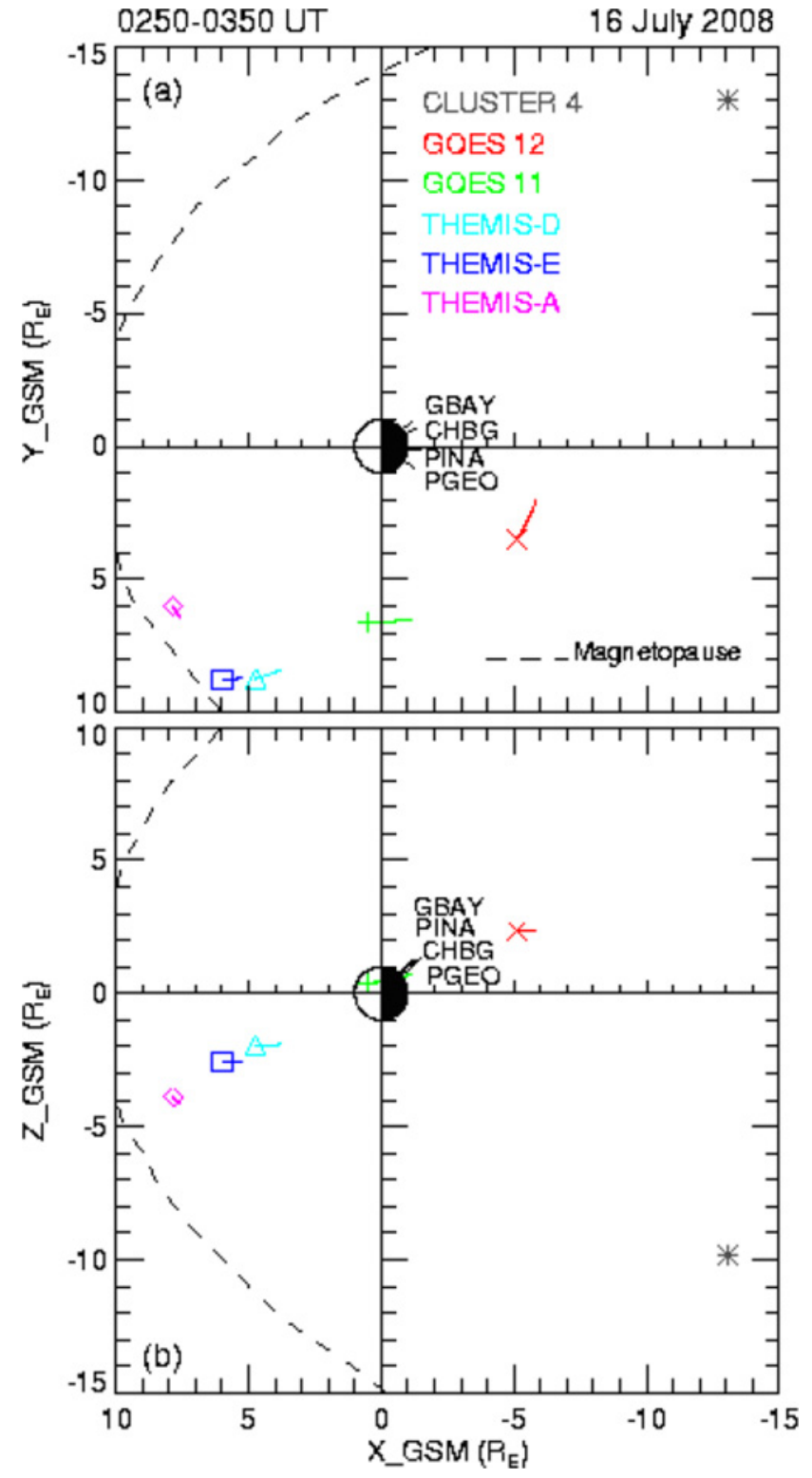

Fig. 8. The locations of THEMIS-A, THEMIS-D, THEMIS-E, GOES 11, GOES 12, and CLUSTER 4 in GSM (a) X-Y and (b) X-Z coordinates from 02:50 UT to 03:50 UT on 16 July 2008. The four THEMIS ground stations at mid $L$ and the nominal magnetopause (dashed trace) are marked for reference.

of two Pi2 bursts for each substorm through the spectral analysis in the aforementioned subsection.

\subsection{Geostationary observations}

In this subsection, we examine if the magnetic variations affected by the SCW formation occurred at geostationary orbit at the onset time of ground $\mathrm{Pi} 2$ bursts. From 02:30 UT to 04:00 UT, GOES 11 moved from the afternoon sector into the dusk sector in the time corresponding to $17: 30 \mathrm{LT}$ to
19:00 LT. Also, GOES 12 orbited into the pre-midnight sector in the time corresponding to 21:30 LT to 23:00 LT. The detailed locations of GOES 11 and GOES 12 can refer to Fig. 8. In Fig. 8, the dashed trace denotes the nominal magnetopause for reference. Their magnetic data with a time resolution of $0.512 \mathrm{~s}$ are used to compare to ground observations. The magnetic field at GOES 11 and GOES 12 is defined as: $H p$, perpendicular to the satellite orbital plane (or parallel to the Earth spin axis in the case of a zerodegree inclination orbit); $\mathrm{He}$, perpendicular to $H p$ and directed earthward; and $H n$, perpendicular to $H p$ and directed eastward. From Fig. 9, one can see that magnetic disturbances at GOES 11 and GOES 12 simultaneously occurred at the $\mathrm{F} 1$ and $\mathrm{S} 2$ onsets. As for the F2 onset, magnetic disturbances first appeared in the $H n$ component and later simultaneously intensified in the three components at both GOES satellites. For the S1 onset, one may argue that the magnetic variations seen by both GOES satellites around the onset time are not discernible from the background level. By close inspection, one cannot deny there were clear wave-like perturbations in the three components at GOES 12 in contrast to those in the $\mathrm{He}$ and $\mathrm{Hn}$ components at GOES 11. The vertical dashed lines in Fig. 9 denote the onset time of ground Pi2s. The top three panels of Fig. 9 show that magnetic disturbances at GOES 11 varied with a larger magnitude at the second onset than that at the first onset for each substorm. From Fig. 9d-f, one can note that GOES 12 observed two strong magnetic fluctuations like substorm-related dipolarizations at the F1 and F2 onsets. As for the last two onsets, the magnetic field at GOES 12 seems to have larger amplitude of wavelike fluctuations as expected to be affected by the SCW at the $\mathrm{S} 1$ onset than that at the $\mathrm{S} 2$ onset. Figure $9 \mathrm{f}$ also shows that the magnitude of the $H p$ component at GOES 12 was enhanced at a time delay to the first onset and was sustained throughout the end of the second substorm time. The $\mathrm{He}$ and $H n$ components varied simultaneously with each $\mathrm{Pi} 2$ onset in Fig. 9d and e. One can also see from Fig. 9b and e that the $H n$ component had a wave-like fluctuation after each $\mathrm{Pi} 2$ onset. Except for the F2 onset at GOES 12 shortly followed by a decrease in $H n$, the magnitude of the $H n$ component increased after three other onsets. According to Sakurai and McPherron (1983), the $H n$ or east-west component increases in the west side of the upward field-aligned current in the left wing of the SCW and decreases in the east side. Hence, magnetic disturbances at geostationary orbit resemble the one affected by the upward field-line current in the left wing of the SCW. These observational results are consistent with the above inference on the SCW formation using ground magnetometer data during the time of interest.

\subsection{Energetic particle injections}

From Fig. 9b and e, one may note that at the $\mathrm{S} 1$ onset, the $H n$ component at GOES 11 and GOES 12, had Pc-like fluctuations and not impulsive substorm-related ones. One may 


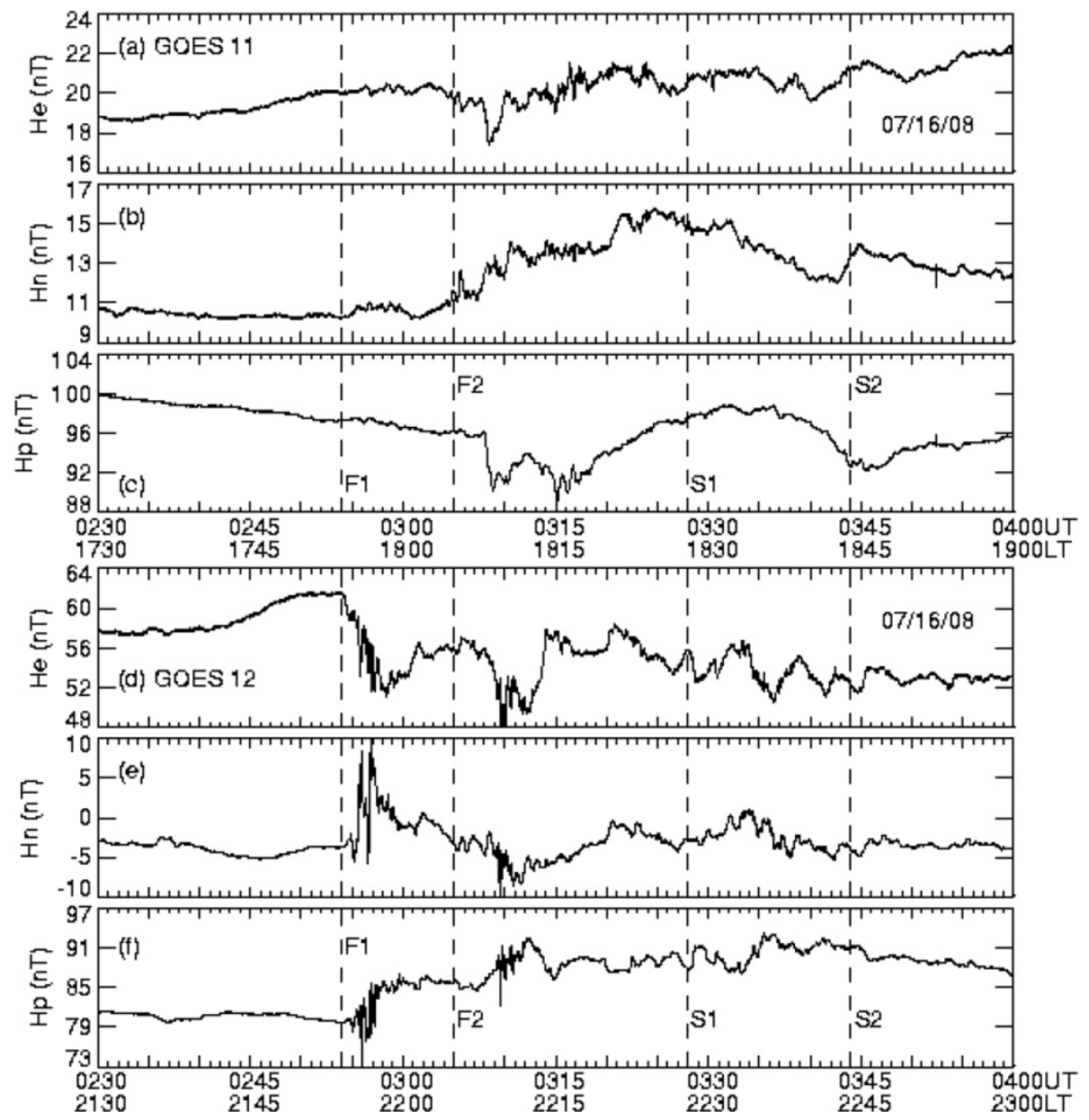

Fig. 9. (a)-(c) The $H e, H n$ and $H p$ components at GOES 11 from 02:30 UT to 04:00 UT on 16 July 2008. (d)-(f) In the same format as Fig. 9a, except for GOES 12. The vertical dashed lines denote ground Pi2 onsets.

argue that they can be induced by the convective electrojets flowing from dawn toward midnight. In this subsection, we further scan the energetic particle measurements by THEMIS probes to see if there was other supporting evidence for the SCW formation, such as energetic particle injections from the magnetotail at each onset. From Fig. 8, one can see that except for THEMIS-A in the afternoon sector, THEMIS-D and THEMIS-E moved close to the predusk flank from 02:50 UT to 03:50 UT. Figure 10 shows the spectral plots of energetic electron fluxes detected by THEMIS-A, THEMIS-D and THEMIS-E. In the same format as Fig. 10, Fig. 11 shows energetic ion fluxes. Noteworthy in Fig. 10, the energetic electron fluxes become enhanced after each ground Pi2 onset. Upon close inspection, the first two enhancements in the energetic electron fluxes are almost simultaneously observed by THEMIS-D, THEMIS$\mathrm{E}$ and THEMIS-A at $\sim 02: 57 \mathrm{UT}$ with a delay time $\sim 3 \mathrm{~min}$ from the F1 and F2 onsets. The enhanced flux intensity seems the largest at THEMIS-D, moderate THEMIS-E and the smallest THEMIS-A. The flux intensity at the S1 and S2 onsets was stronger than that at the F1 and F2 onsets. The delay time for S1 and S2 is shorter than that for F1 and F2. Likewise, Fig. 11 shows that the enhancement of energetic ion fluxes followed by the onset of ground Pi2s. For the enhancement in the energetic ion fluxes, the delay time at F1 is $\sim 5$ min longer than $\sim 3$ min for the energetic electron fluxes. By contrast, the energetic ion flux first appeared at THEMIS$\mathrm{D}$ at $\sim$ 03:00 UT and spread to THEMIS-E at $\sim 03: 05$ UT and then to THEMIS-A at 03:07 UT. At the F2 onset, THEMISD detected a stronger intensity at $\sim 03: 07$ UT that was simultaneously observed by THEMIS-E and THEMIS-A with a delay time $\sim 2 \mathrm{~min}$. As for the S1 and S2 onsets, the energetic ion fluxes seem to increase prior to the ground Pi2 onsets. This may be due to the late energetic particle flux enhancements caused by the first substorm. Consequently, THEMIS-D first observed four consecutive enhancements of energetic electron fluxes and later they were subsequently seen by THEMIS-E and THEMIS-A. Since energetic particle injection is a well-known signature for substorm onset (e.g. Baker et al., 1996), their source can be substorm-related and 


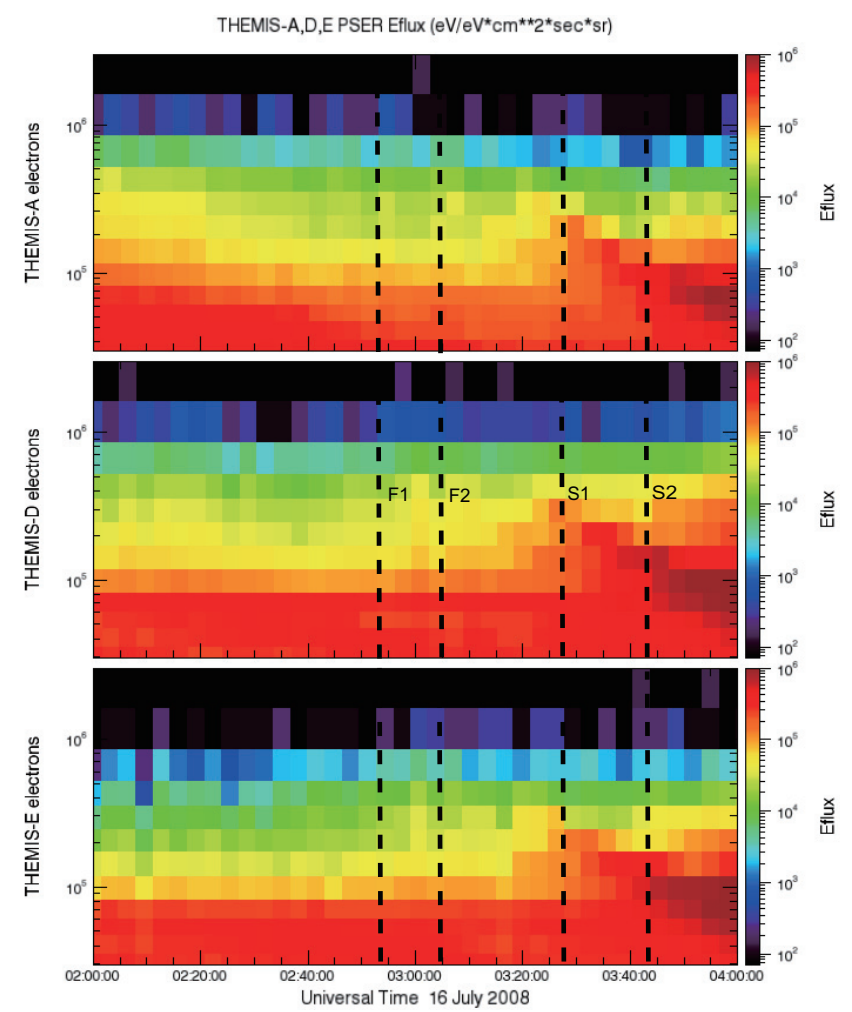

Fig. 10. Energetic electron injections observed by THEMIS-A, THEMIS-D and THEMIS-E probes from 02:00 UT to 04:00 UT on 16 July 2008. The vertical dashed lines denote ground Pi2 onsets.

located at the near-Earth magnetotail. These THEMIS probe observations indicate that for the two pairs of onsets, magnetic disturbances at geostationary orbit can be affected by the SCW formation resulting from energetic electron injections similar to those at substorm onsets.

\subsection{Horizontal magnetic variation vectors}

To justify the link of ground Pi2s to the SCW formation, we have plotted the horizontal magnetic variation vectors consisting of $\mathrm{H}$ - and $\mathrm{D}$-components, removed from their $12 \mathrm{~min}$ running means, sensed by all the available THEMIS observatories to simulate the equivalent current vector like those adopted by earlier studies (e.g. Glassmeier, 1987; Untiedt and Baumjohann, 1993). Figure 12 shows the horizontal magnetic variation vectors in corrected geomagnetic latitude versus corrected geomagnetic longitude at each $\mathrm{Pi} 2$ onset. The black circles denote the locations of the ground stations. The dot-centered circle denotes the assumed location of the upward field-aligned current and the cross-centered circle the downward field-aligned current. At 02:55 UT (corresponding to the F1 onset), there are two magnetic vortex patterns of which the first located at CGM lat. $60.0^{\circ}$ and CGM longitude $360^{\circ}$ resembles the one affected by the downward field-aligned current and the other about CGM lat. $65.0^{\circ}$ and

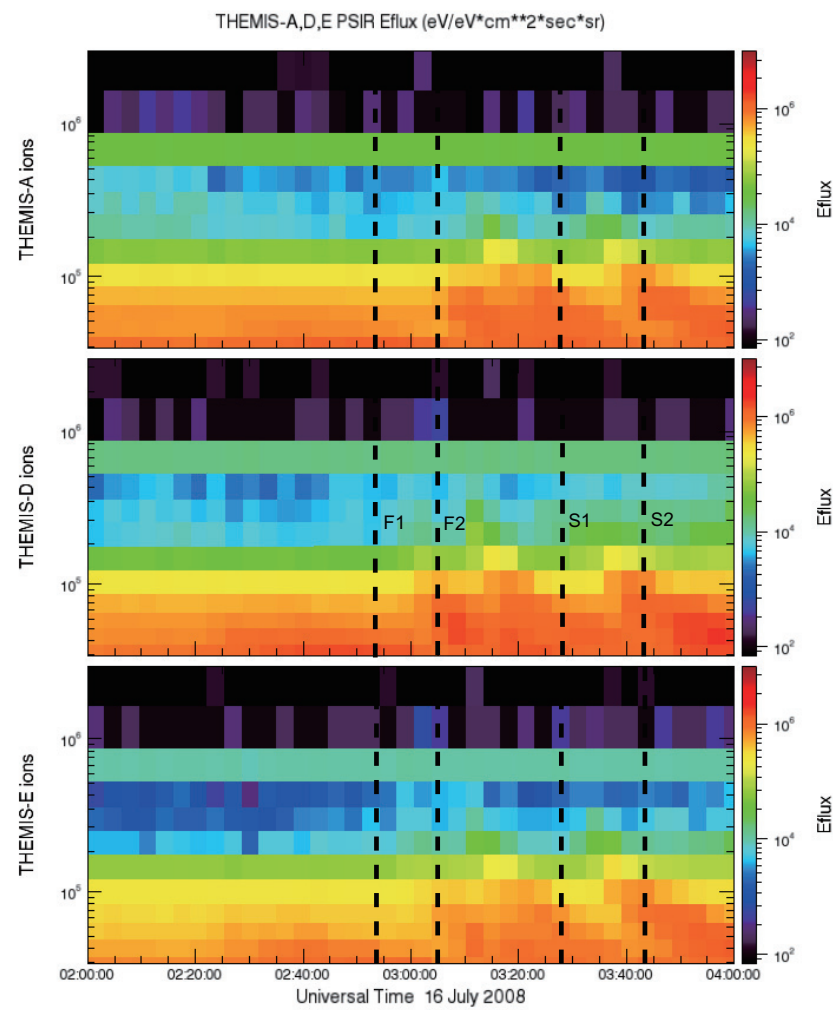

Fig. 11. In the same format as Fig. 10, except for energetic ion injections.

CGM longitude $310^{\circ}$ by the upward field-aligned current. As for the F2 onset at 03:08 UT, the magnetic vortex pattern becomes the one dominantly affected by the downward field-aligned current. The top right panel of Fig. 12 shows the magnetic vortex pattern at the S1 onset $~ 03: 29$ UT like the one dominated by the upward field-aligned current. As for the S2 onset at 03:45 UT, the magnetic vortex pattern does not seem strong compared to those at three other onsets, but does resemble the one affected by the downward field-aligned current. As a result, the plots of the horizontal magnetic variation vectors once again manifest that, for this event, the ground Pi2 onsets are closely tied to the SCW formation.

\subsection{Hodogram analysis}

Earlier studies (e.g. Lester et al., 1983, 1984, 1989) suggested that mid-latitude Pi2s have the characteristic polarization pattern affected by the SCW formation. In this subsection, we have performed the hodogram analysis of THEMIS Pi2s at mid $L$ at a longitudinal separation to further verify their link to the SCW formation. The ground hodogram includes the perturbed $\mathrm{H}$ - and $\mathrm{D}$-components. The hodogram pattern is identified as clockwise $(\mathrm{CW})$ polarization and counter-clockwise $(\mathrm{CCW})$ polarization along the direction of the ambient magnetic field. Figure 13 shows the 


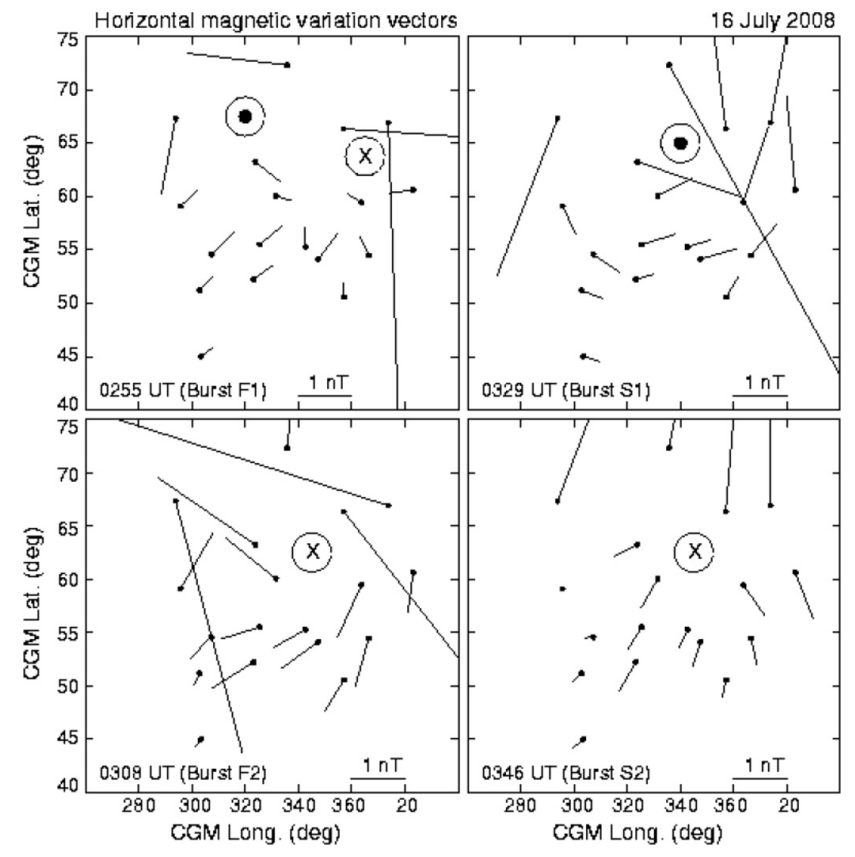

Fig. 12. The horizontal magnetic variation vectors related to ground Pi2 onsets on 16 July 2008. The dot-centered circle denotes the assumed location of the upward field-aligned current and the crosscentered circle the downward field-aligned current.

wave polarizations at the mid- $L$ THEMIS stations at four different longitudes. At the top of each hodogram column is the corrected magnetic longitude of the mid- $L$ THEMIS stations. One can see from Fig. 13 that the wave polarization is $\mathrm{CCW}$ at the mid- $L$ stations for each $\mathrm{Pi} 2$ burst. Moreover, their polarization pattern is the same as one another and polarized with the direction of the major axis toward the SCW center. These observational results are consistent with Fig. 1 of Lester et al. (1984).

\subsection{AL index and IMF observations}

To verify if four consecutive onsets are correlated with one another in a series and externally triggered, we have also looked at the AL index provided by the World Data Center for Geomagnetism at Kyoto University in Japan and the IMF observations just in front of the Earth's magnetopause. Figure 14 shows the AL index, the IMF clock angle, the IMF $B_{\mathrm{y}}$, and the IMF $B_{\mathrm{z}}$ sensed by the ACE satellite in the upstream region shifted to the location of the Earth at $\sim 1 \mathrm{AU}$ (corresponding to XGSM $\sim 17.0 R_{\mathrm{E}}$ ) and by THEMIS-B at XGSM $\sim 25.0 R_{\mathrm{E}}$ from 02:00 UT to 04:00 UT on 16 July 2008. Details of instrumentation and measurements of the fluxgate magnetometer on board THEMIS-B can be found in Auster et al. (2008). In this study, growth and decay mean decrease and increase in the AL index, respectively. From Fig. 14a, one can see two distinct periods of AL variations of growth-to-decay during the occurrence time of four

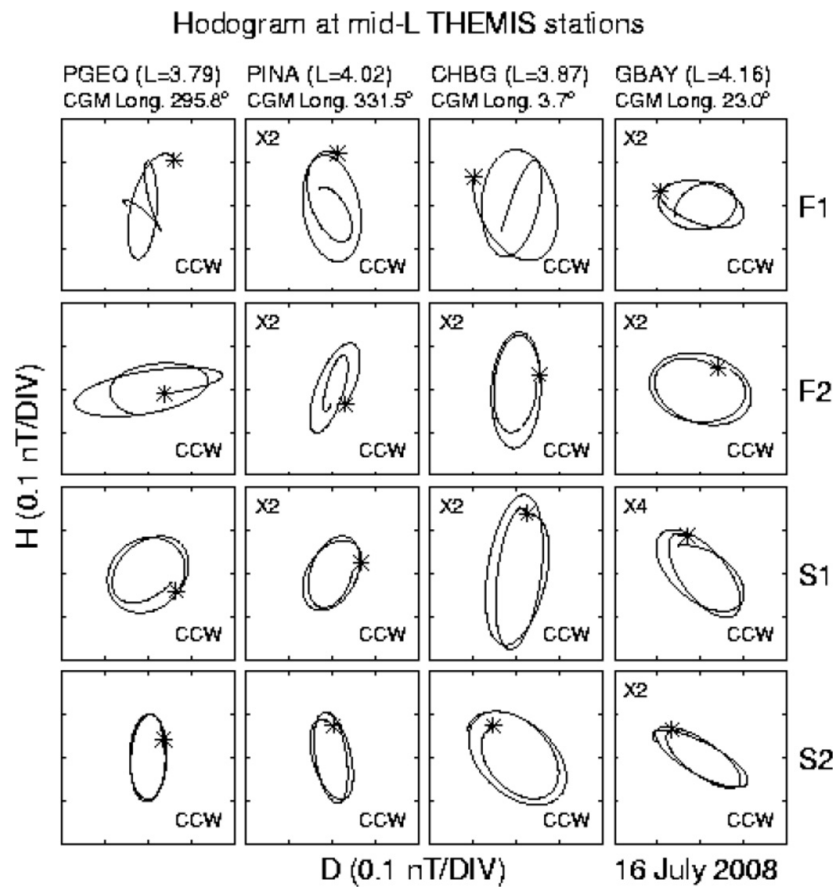

Fig. 13. The hodogram of four Pi2 bursts at four mid- $L$ THEMIS stations. The asterisk denotes the starting point. CCW denotes counterclockwise polarization.

Pi2 bursts. The horizontal solid (dotted) line marks the time interval of growth (decay) in AL magnitude. The first growth began at $\sim 02: 54$ UT and continued to $\sim 03: 14$ UT followed by decay to $\sim 03: 22$ UT. The second growth was from $\sim 03: 22$ UT to $\sim 03: 38$ UT and then decay from $\sim 03: 38$ UT to $\sim 03: 48$ UT. In other words, there are two similar variation cycles of growth-to-decay for both periods. According to Rostoker (2002), a substorm activity can be recognized from the growth and subsequent decay of the AL index. Hence, a pair of ground Pi2 bursts occurring in a cycle of growthto-decay in the AL index can be regarded as two onsets in a single substorm. By mapping the $\mathrm{Pi} 2$ onset timing to the satellite observations, one can find from Fig. 14 that the IMF variations in the upstream region shifted to $\sim 1 \mathrm{AU}$ appear to be the same as observed by THEMIS-B during the time of interest. Since the magnitude of the IMF $B_{\mathrm{y}}$ stayed around $4 \mathrm{nT}$ larger than that of the IMF $B_{\mathrm{Z}}$ less than $2 \mathrm{nT}$, the IMF clock angle remained low, $90^{\circ}$, at each onset. It is worth noting that in Fig. 14d and g, there are two distinct IMF variation cycles of north-to-south and north again for both double-onset substorms. For the first variation cycle, the IMF remained horizontal until 02:35 UT and afterwards turned southward through the F1 onset and then had northward turning prior to the F2 onset. As for the second variation cycle, the S1 and S2 onsets occurred as the IMF stayed south and followed by a northward turning. The growth in AL magnitude prior to $\mathrm{S} 1$ began right at the end of decay in AL magnitude after F2. This may be due to a short excursion time of the IMF 


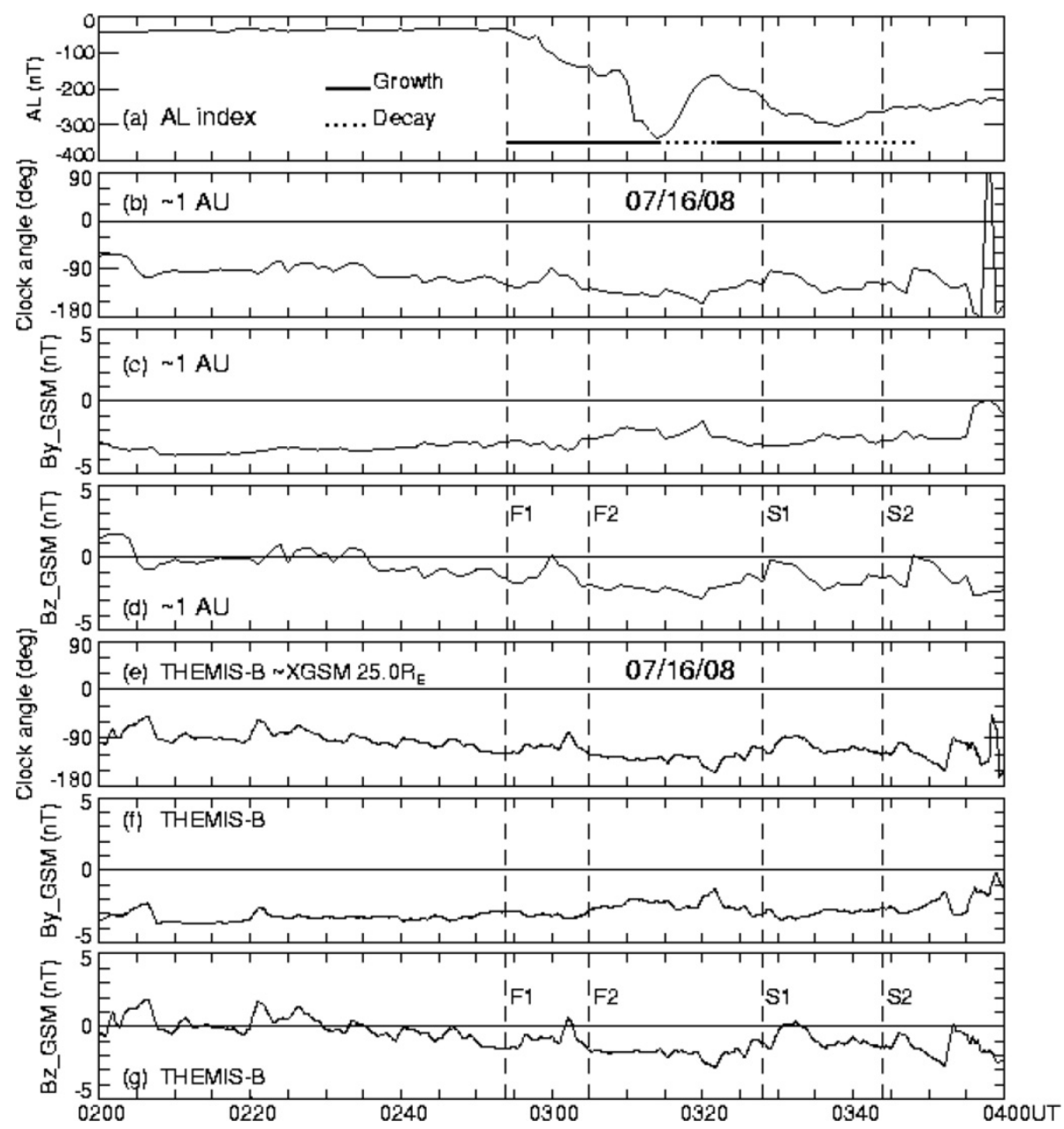

Fig. 14. The AL index, the IMF variations at $\sim 1 \mathrm{AU}$ (corresponding to $\mathrm{XGSM} \sim 17.0 R_{\mathrm{E}}$ ) and THEMIS-B at $\mathrm{XGSM} \sim 25.0 R_{\mathrm{E}}$ related to ground Pi2 onsets on 16 July 2008. The horizontal solid (dotted) line marks the time interval of growth (decay) in AL magnitude.

turning northward. Without comparison to AL, one may argue that the IMF variation cycle for the last two onsets can be described as south-to-north and then south. According to Russell (2000), double substorm onsets can occur when the IMF undergoes a variation cycle of north-to-south and north again. Moreover, the first onset appears in a period time after the IMF switches to south from north. Hence, we suggest the IMF variation cycle for S1 and S2 to be north-to-south and north again.

As for the AL variation shown in Fig. 14a, one may note the lack of a subsequent growth after the S2 onset opposite to the enhanced growth post the F2 onset. Moreover in Fig. 14g, the IMF sensed by THEMIS-B has been southward for $\sim 10$ min prior to the $\mathrm{S} 2$ onset in contrast to the case of the $\mathrm{F} 2$ onset with a relatively short time that can be not long enough to inhibit the further onset. One may hereby argue that the S2 onset could be not the Russell-model type. Lyons et al. (1997) suggested that the mean delay time between magnetopause contact and an IMF trigger and substorm on- set is $\sim 9$ min. Since the solar wind speed was $\sim 570 \mathrm{~km} \mathrm{~s}^{-1}$ during the time of interest, the IMF propagation time from THEMIS-B at XGSM $\sim 25.0 R_{\mathrm{E}}$ to the Earth's magnetopause is $\sim 3 \mathrm{~min}$. In other words, the time interval $\sim 10 \mathrm{~min}$ of the IMF turning southward is not longer than $\sim 12$ min to suppress the S2 onset. Hence, it is plausible that the S2 onset can be explained by the Russell (2000) model.

According to Russell et al. (2003), the maximum reconnection rate can occur for a low clock angle that is away from due south. In addition, Grocott et al. (2005) reported SuperDARN observations of the nightside ionospheric flows as a result of reconnection in an asymmetric tail under influence of IMF $B_{\mathrm{y}}$. Hence with mapping of the Pi2 onset timing, the observational results at the subsolar magnetopause and THEMIS-B suggest that double-onset substorms can be externally triggered in association with the IMF variations. 


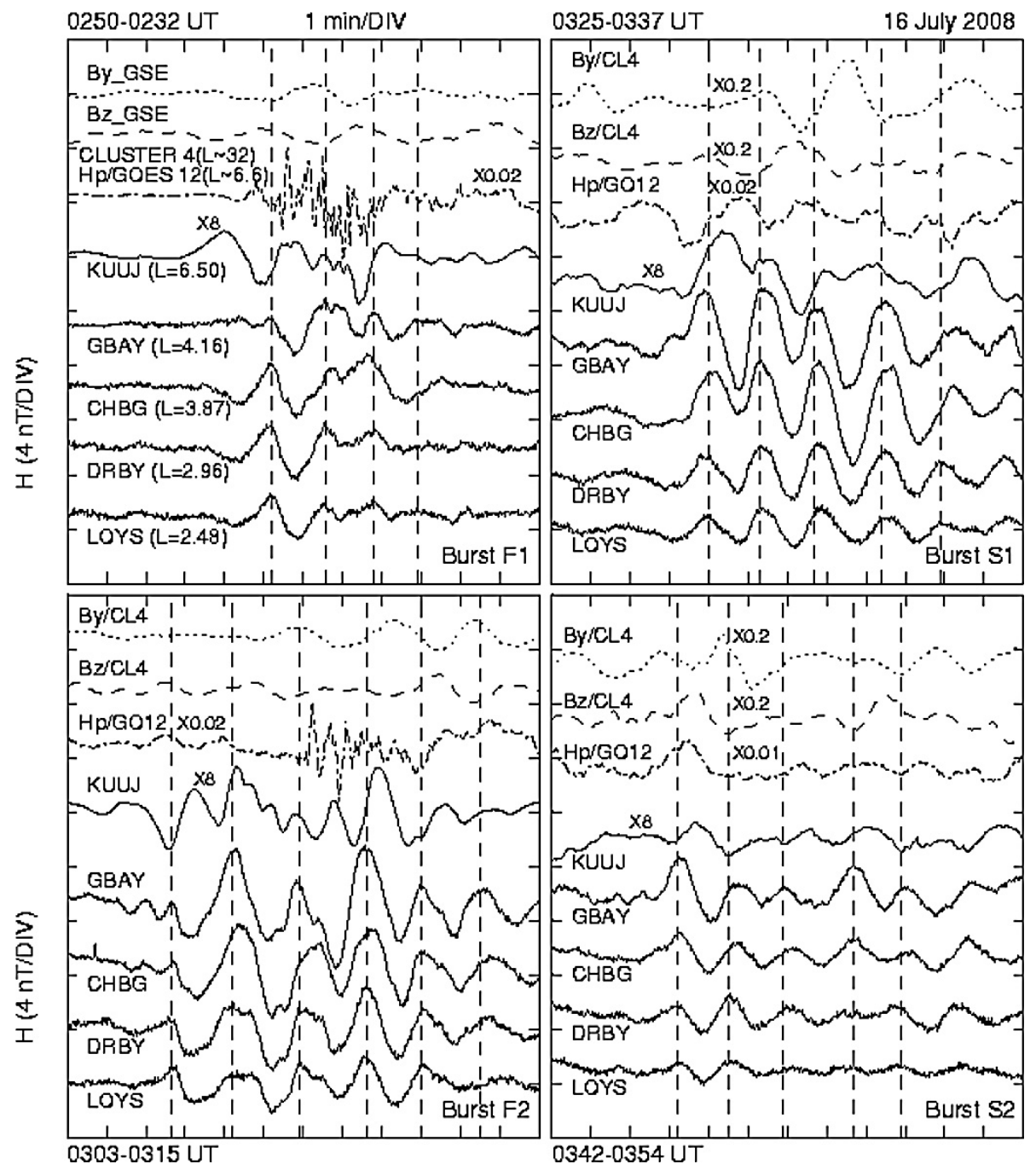

Fig. 15. The waveforms at the THEMIS ground stations along the East Coast of North America for four Pi2 bursts compared to magnetic fluctuations sensed by GOES 12 and CLUSTER 4 satellites. The solid traces denote the H-component at the THEMIS ground stations. The dot-dashed trace denotes the $H p$ component at GOES 12. The dotted and dashed traces denote the By_GSE and Bz_GSE components at CLUSTER 4, respectively. The vertical dashed lines denote the most same peaks in the waveforms seen by the THEMIS ground stations along the East Coast.

\section{Discussion}

As mentioned in the introduction, previous studies by Cheng et al. (2002a, b) discovered the association of substorm onsets with the IMF variations by using only consecutive Pi2 bursts. For a typical substorm process in the near-Earth magnetotail as Baker et al. (1996) pointed out, the SCW formation, auroral breakups and magnetic bays at high latitudes are the common hydromagnetic disturbances in addition to Pi2 pulsations. More ground and space observations (e.g. Mishin et al., 2001; Cheng et al., 2005) demonstrated that nightside disturbances can have two or more onsets during a substorm sequence. However, these early studies did not have well-coordinated measurements for further verifications of the link of ground Pi2s with other substorm signatures in the nightside magnetosphere. Thus by adopting multipoint observations by the THEMIS mission, the present study is more comprehensive than early studies examining the relationship between multiple onsets of nightside substorm-related disturbances and the IMF variations. More importantly, the 16 July 2008 event demonstrates an unprecedented global picture of the dynamic process of two double-onset substorms in response to two distinct IMF variation cycles of north-to-south and north again.

In this study, two pairs of Pi2 bursts appeared simultaneously at the available THEMIS ground observatories from 02:50 UT to 03:50 UT on 16 July 2008 (see Figs. 23 ). Based upon the same dominant frequency band from 


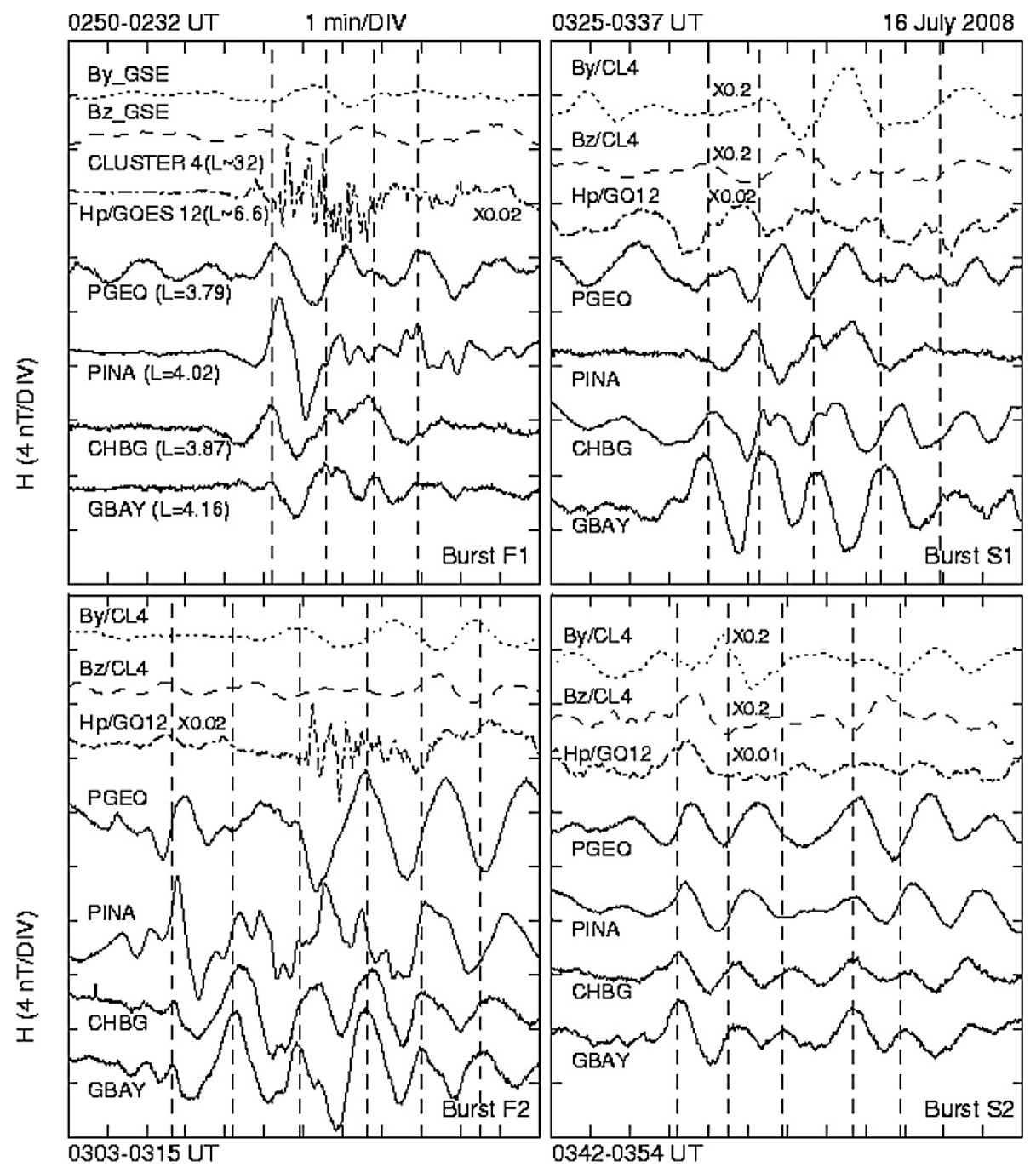

Fig. 16. Waveforms for the mid- $L$ stations near $L=4$. Comments of caption to Fig. 15 apply.

the wavelet transformation and the same wave phase among THEMIS ground stations, four $\mathrm{Pi} 2$ bursts are identified as a fast magnetospheric cavity mode and not as a plasmaspheric cavity mode. To further justify the above inferences, we have also performed the waveform comparison at the stations around the East Coast of North America by removing the ambient magnetic field. Likewise, the magnetic fluctuations sensed by GOES 12 and CLUSTER 4 satellites are included to compare to those at the ground stations. Figure 15 shows the waveforms of $\mathrm{H}$-component (solid trace) at the ground stations along the East Coast, the $\mathrm{Hp}$ component (dot-dashed trace) at GOES 12, and the By_GSE (dotted trace) and $B z_{-} G S E$ (dashed trace) components at CLUSTER 4. The vertical dashed lines denote the most same peaks in the waveforms at the ground stations along the East Coast. Note that the amplitude of Pi2s at KUUJ $(L=6.50)$ is 8 times larger than those at lower latitudes. The magnetic data at GOES 12 are low-pass filtered with running mean in $40 \mathrm{~s}$ in- terval and amplified 50 times for the first three bursts and 100 times for the last burst. For each Pi2 burst along the East Coast, the waveforms at KUUJ ( $L=6.50)$ seem out of phase with those at the stations at lower latitudes. From GBAY ( $L=4.16)$ down to LOYS $(L=2.48)$, their waveforms are almost in the same phase.

Like the waveforms at KUUJ ( $L=6.50$ ), the magnetic fluctuations at GOES 12 seem out of phase with those at the stations at lower latitudes for the most same peaks of each ground Pi2 burst. Moreover, for the F1 burst, magnetic fluctuations at GOES 12 had a period of $\sim 24 \mathrm{~s}$ from 02:55 UT to 02:58 UT look like those at KUUJ $(L=6.50)$. But for the F2 burst, KUUJ ( $L=6.50)$ observed the same higher frequency of magnetic pulsations occurring $2 \mathrm{~min}$ prior to those at GOES 12 from 03:09 UT to 03:12 UT. The dominant period of ground Pi2s is $\sim 100 \mathrm{~s}$ and the location of GOES 12 is beyond the nominal plasmapause. Since the pulsation amplitudes at $\operatorname{KUUJ}(L=6.50)$ are much larger than those at lower 


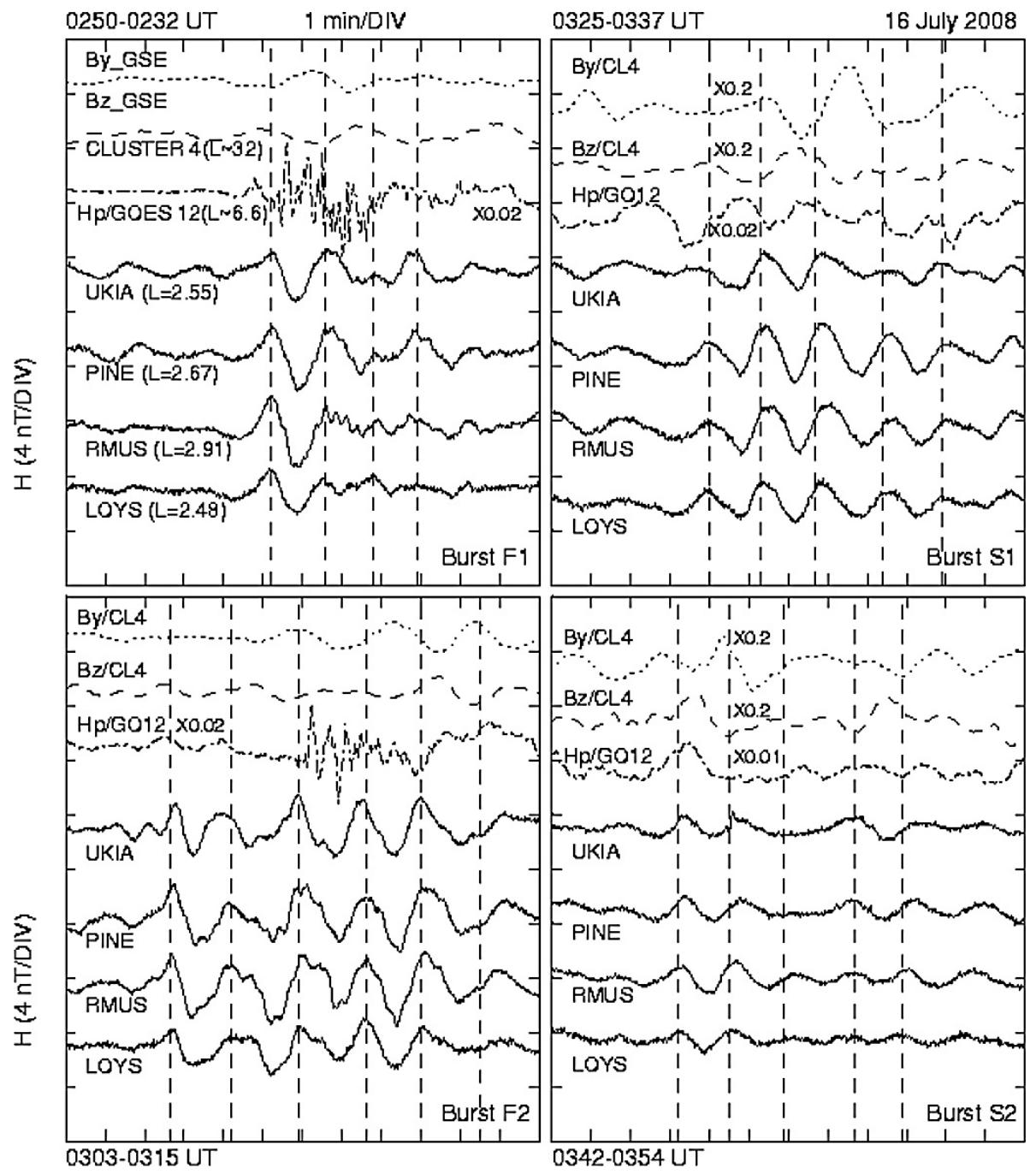

Fig. 17. Waveforms for the low $L$ stations. Comments of caption to Fig. 15 apply.

$L$ stations, their source can be located at the magnetotail beyond the plasmapause. Moreover like those at GOES 12, the waveforms at KUUJ $(L=6.50)$ seem more perturbed with broad band than those at lower $L$ stations. This feature is consistent with the broader frequency extent of the power enhancements at KUUJ $(L=6.50)$ than other lower $L$ stations (see Figs. 4-6). In the same format as Fig. 15, Figs. 16 and 17 respectively shows the waveforms of $\mathrm{H}$-component at the mid- $L$ stations near $L=4$ and the low $L$ stations. By contrast, the waveforms of Pi2s at mid- $L$ and low $L$ stations are almost in the same phase as those along the East Coast except for PINA $(L=4.02)$ and RMUS $(L=2.91)$ having a shorter period for the F1 burst only. These are consistent with the observational results by Cheng et al. (2009a), that THEMIS Pi2s have the characteristics of the fundamental magnetospheric cavity mode resonances with $180^{\circ}$ of phase change from high to low latitude across the plasmapause (see Lin et al., 1991; Cheng et al., 1998, 2000). Hence, consec- utive $\mathrm{Pi} 2$ bursts for this event can be a fast magnetospheric cavity mode.

During the time of interest, CLUSTER 4 was located at about $(-13.0,-12.0,-11.0) R_{\mathrm{E}}$ in GSM coordinates corresponding to be in the south lobe (see Fig. 8 for the locations). The magnetic data at CLUSTER 4 are low-pass filtered with a frequency less than $25 \mathrm{mHz}$. From Fig. 15, one can find that By_GSE and Bz_GSE components have wavelike fluctuations at each ground Pi2 onset. By contrast, the fluctuation amplitude in the $B y_{-} G S E$ component seems larger than that in the $B z_{-} G S E$ component. The trend of the fluctuation amplitude in the By_GSE component looks similar to those on the ground. Since CLUSTER 4 was closer to dawn than to midnight, one may argue that it can be affected by the large scale convection electrojet in the morning sector. We found that the IMAGE magnetometer stations were close to the meridian of its footpoint. Via the website (http://www.geo.fmi.fi/image), one can see that the IU index 
(corresponding to $\mathrm{AU}$ ) had the wave-like variations similar to those seen by THEMIS stations at each Pi2 onset. Their amplitudes are also smaller than those of THEMIS Pi2s. This suggests that their sources can be located around midnight and not dawn. Since By_GSE and Bz_GSE can represent the transverse and compressional components respectively, the wave-like magnetic fluctuations in the south lobe can be field line oscillations in the mode of shear Alfvén waves driven by fast waves resulting from the impulsive source in the nearEarth magnetotail. For this event, the duration of ground Pi2s is about 6-12 min longer than the travel time between the auroral ionosphere and their presumed source site in the magnetotail. It is well known (e.g. Russell and McPherron, 1973) that the near-Earth impulsive source in the magnetotail can be as close as $\sim 10 R_{\mathrm{E}}$ from the Earth. If the Alfvén speed is $\sim 1000 \mathrm{~km} \mathrm{~s}^{-1}$, the travel time between auroral ionosphere and the presumed source site could be $2-3 \mathrm{~min}$, much less than 6-12 min determined from the duration time of ground Pi2 bursts. For example in Fig. 2b, the D-component at GBAY apparently shows the longest duration time $\sim 12 \mathrm{~min}$ at the S1 onset and the shortest one $\sim 6 \mathrm{~min}$ at the S2 onset. Thus, shear Alfvén waves bouncing back along the open field lines in between the auroral ionosphere and the tail lobes can drive field line oscillations to form standing waves in the duration time of their source. Since the plasmapause is not a fixed boundary, not all fast waves from the tail are trapped in the plasmaspheric cavity. During their duration time, fast waves bouncing between their tail source site and the Earth can be transmitted through the plasmapause as a node to excite a magnetospheric cavity resonance mode. Hence, it is reasonable to infer their source location with frequency comparison of both Pi2s for each substorm.

In this study, the wave period of Pi2s can be identified with a time interval between the most similar adjacent peaks in the $\mathrm{H}$-component seen by ground stations along the East Coast. In addition to the distinct $\mathrm{Pi} 2$ period shown in Fig. 6, one can find from Fig. 15 that they are about $60 \mathrm{~s}, 72 \mathrm{~s}$ and $84 \mathrm{~s}$ for the F1 burst. For the F2 burst, the wave periods are about $84 \mathrm{~s}, 90 \mathrm{~s}, 102 \mathrm{~s}$ and $108 \mathrm{~s}$. The wave periods of the S1 bursts are about $74 \mathrm{~s}, 84 \mathrm{~s}, 90 \mathrm{~s}$ and $102 \mathrm{~s}$. For S2 burst, the wave periods are about $74 \mathrm{~s}, 78 \mathrm{~s}, 84 \mathrm{~s}$ and $108 \mathrm{~s}$. Statistically, their wave periods are $72.0 \pm 12.0 \mathrm{~s}$ for the F1 burst, $96.0 \pm 10.9 \mathrm{~s}$ for the F2 burst, $87.5 \pm 11.7 \mathrm{~s}$ for the S1 burst and $86.0 \pm$ $15.2 \mathrm{~s}$ for the S2 burst, respectively. Despite having no fixed discrete periods, they are consistent with the conclusion from the wavelet transformation shown in Figs. 4-5 that the same dominant frequency band is centered at $0.01 \mathrm{~Hz}(\sim 100 \mathrm{~s})$.

According to Takahashi et al. (2003), two harmonic frequencies of Pi2s are $\sim 7 \mathrm{mHz}(\sim 142.8 \mathrm{~s})$ and $\sim 12 \mathrm{mHz}$ $(\sim 83 \mathrm{~s})$ with comparison of the ground data at Kakioka ( $L=1.26)$ to the CRRES observations around the plasmapause. Although the second harmonic frequency is the same as the one sensed by THEMIS ground stations, Takahashi et al. argued that they result from plasmaspheric cavity resonances and not from a fast magnetospheric cavity mode as we suggested. The other difference is that their phase relation is determined by comparison of one ground station to single-satellite observations rather than using more ground data from an array like the THEMIS observatories. Moreover from Figs. 16 and 17, one can see that the magnetic fluctuations at PINA ( $L=4.02)$ and RMUS $(L=2.91)$ look like those seen by CRRES in space and not sensed by Kakioka ( $L=1.26)$ on the ground. As for these similar magnetic perturbations at THEMIS ground stations, the waveform and wave period $\sim 29$ s of the pulsations at PINA $(L=4.02)$ are close to those seen by GOES 12 but different from those at RMUS $(L=2.92)$ with a shorter period $\sim 14 \mathrm{~s}$. This implies that some higher frequency pulsations are not global phenomena and may be caused by other localized sources. Thus for lack of satellite observations in the inner magnetosphere, it remains unknown why $\mathrm{Pi} 2$ bursts for this event occur in a different wave mode from those studied by Takahashi et al. (2003).

Moreover, the mapping of ground $\mathrm{Pi} 2$ onset timing to the IMF observations shows that they successively appear in a pair under two variation cycles of north-to-south and then north (see Fig. 14). The wavelet transformation of $\mathrm{H}$ - and D-components at the stations along the East Coast of North America shows that the dominant frequency band of the first Pi2 onset seems higher than that of the second onset for each substorm (see Figs. 4-5). According to Russell (2000), the interplay between near-Earth and distant neutral points in the magnetotail can lead to two onsets as the IMF undergoes a single variation cycle of north-to-south and to north again. The first onset occurs when the IMF turns southward for a time period and reconnection at the near-Earth neutral point first begins on closed field lines within the plasma sheet which can map to lower latitudes below the auroral zone. The other onset commences when the IMF has northward turning and the near-Earth neutral point reaches the open flux of the tail lobes mapping to higher latitudes over the auroral zone. This suggestion is consistent with the INTERMAGNET magnetograms on 16 July 2008 that aurora-related electrojet at the first onset occur at lower latitudes than those for the second onset in each cycle (see Fig. 7).

For this event, no observations in the inner plasma sheet are available for testing the inference from ground observations that at the second onset magnetotail reconnection reaches the lobes. One may argue that two pair of Pi2 bursts can be associated with four substorms and not two doubleonset substorms instead. According to Rostoker (2002), substorm activity can be recognized from the growth and subsequent decay of the AL index. Hence, we examine the AL index to see if there is the geomagnetic activity in response to the dynamic change resulting from magnetic reconnection at the near-Earth neutral point during successive substorms. It is noteworthy in Fig. 14a that there are two similar variation cycles of growth-to-decay and growth again right after the beginning of the decay for both periods. Since the onset of the second growth is close to the beginning of decay, 
they can be regarded as double onsets in a single substorm. Namely, the second growth can be interpreted as a signature of magnetotail reconnection reaching the lobes. Hence, this further justifies that four Pi2 bursts can be regarded as the occurrence indicators of two double-onset substorms.

Alternatively, the $H p$ component at GOES 12 moving in the pre-midnight sector was enhanced after the F1 onset and was sustained through the S2 onset (see Fig. 9f). These magnetic disturbances at geostationary orbit are different from the other types of geomagnetic activity in relation to the current disruption. Without in-situ observations tailward of geostationary orbit, we cannot rule out the possibility that the current disruption could occur elsewhere and not be seen by GOES 12 during the time of interest. For the F1 and F2 onsets, however, ground Pi2 waveforms clearly appeared prior to enhanced $H p$ variations sensed by GOES 12 (see Figs. 9f and 15) affected by the SCW formation. For the two pair onsets, THEMIS-D orbiting to the dusk flank first detected four consecutive enhanced energetic electron and ion fluxes that later measured by THEMIS-E and THEMIS-A in the afternoon sector (see Figs. 10-11). Moreover, the horizontal magnetic variation vectors showed the patterns resemble the ones dominantly affected by the upward field-aligned current at the first onset and by the downward field-aligned current at the second onset for each substorm (see Fig. 12). The hodogram of THEMIS Pi2s at mid $L$ (see Fig. 13) and geostationary observations verify the SCW formation. These observational results, consistent with the early study by Glassmeier et al. (1988), also suggest that the magnetic enhancements at geostationary orbit and on the ground can be caused by the SCW formation. According to Shiokawa et al. (1998), the SCW formation can be due to braking of fast flows or bursty bulk flows resulting from magnetic reconnection in the near-Earth magnetotail. Recently, Kepko et al. (2004) showed that the onsets of both nightside Pi2 pulsations and magnetic bay variations were simultaneous with auroral brightening. They argued that these are driven by the flow bursts in the magnetotail.

Since the duration of fast flows is a few minutes, Kan (1998) proposed a globally integrated substorm model in which fast flows are driven by patchy-bursty 3-D reconnection that appears to be more likely a natural mode of spontaneous reconnection in the magnetotail than single $\mathrm{X}$ line steady 2-D reconnection. However, we do not find such stochastic behavior. To explain the double-onset observations, Russell (2000) improved the near-Earth neutral point model with emphasis on the role of the distant neutral point and how it could play a role in driven substorm triggering. Since nightside substorm-related disturbances observed by the THEMIS mission successively occur during two distinct IMF variation cycles of north-to-south and north again, the 16 July 2008 event can thus be explained with the Russell (2000) model. Namely, this event is consistent with the substorm model of deterministic external triggering by the IMF variations of north-to-south and then north.

\section{Summary and conclusion}

On 16 July 2008, four successive Pi2 bursts occurred simultaneously at the ground-based observatory system for the THEMIS mission. Wavelet transformation shows a new characteristic feature that for each pair at high latitudes the dominant frequency band of the first burst becomes higher than that of the second one. But at low latitudes, their dominant frequency band appears to be unchanged. Both pairs predominantly result from fast magnetospheric cavity waves and with the second burst also containing shear Alfvén waves. INTERMAGNET magnetograms at auroral latitudes showed magnetic variations affected by two recurrent electrojets for each pair. The ground-based magnetometers and geostationary orbit sensed magnetic perturbations resembling the ones affected by the SCW formation. For the two pairs of onsets, four consecutive enhancements of energetic electron and ion fluxes first were detected by THEMIS$\mathrm{D}$ situated in the dusk flank and then subsequently seen by THEMIS-E and THEMIS-A in the afternoon sector. The horizontal magnetic variation vectors, consisting of $\mathrm{H}-$ and D-components, had the vortex patterns similar to the ones induced by the upward and downward field-aligned currents during substorm times. The hodogram at mid- $L$ stations had a polarization pattern consistent with the one induced by the substorm current wedge for each Pi2 burst. These observations display two recurrent occurrences of double-onset substorms.

In the meantime, the THEMIS-B probe at XGSM $\sim 25.0 R_{\mathrm{E}}$ on the sunward side of the magnetosphere observed the same two IMF variation trends with a low clock angle as those in the upstream region shifted to just in front of Earth's magnetopause. The mapping of ground Pi2 onset timing to the IMF observations shows their double recurrences under two variation cycles of north-to-south and then north. The wave-like magnetic fluctuations observed by CLUSTER 4 in the south lobe and expected to be driven by near-Earth reconnection, look similar to ground Pi2 waveforms. In short, this THEMIS event study reveals the plausible link of two recurrences of double-onset substorms to magnetotail reconnection externally triggered by IMF variations.

Acknowledgements. The THEMIS data were obtained via CDAWeb and AIDA at NCU, Taiwan. The CARISMA magnetometer array is operated by the University of Alberta, funded by the Canadian Space Agency. We thank the national institutes that support them and INTERMAGNET for promoting high standards of magnetic observatory practice. The GOES 11, GOES 12 magnetic field data were provided by H. J. Singer at NOAA via CDAWeb. The Kyoto AL index and the IMF data at $1 \mathrm{AU}$ were provided by J. H. King and N. Papatashvilli at NASA GFSC via CDAWeb. The magnetic data at CLUSTER 4 were provided by A. Balogh at ICSTM via CDAWeb. This work was supported by National Science Council of R. O. C. on Taiwan under the grant NSC 98-2111-M-150-001, and by National Space Organization 
under the grant 97-NSPO(B)-SP-FA07-01(A). The work at NCU was supported in part by Ministry of Education under the Aim for Top University Program. The work at UCLA was supported by National Aeronautics and Space Administration under the research grant UCB/NASA 5-02099. The contribution by KHG was financially supported by the German Zentrum fuer Luft-und Raumfahrt and the German Ministerium fuer Wirtschaft und Technologie under grant 50QP0402.

Topical Editor R. Nakamura thanks two anonymous referees for their help in evaluating this paper.

\section{References}

Angelopoulos, V.: The THEMIS Mission, Space Sci. Rev., 141, 534, doi:10.1007/s11214-008-9336-1, 2008.

Auster, H. U., Glassmeier, K. H., Magnes, W., Aydogar, O., Baumjohann, W., Constantinescu, D., Fischer, D., Fornacon, K. H., Georgescu, E., Harvey, P., Hillenmaier, O., Kroth, R., Ludlam, M., Narita, Y., Nakamura, R., Okrafka, K., Plaschke, F., Richter, I., Schwarzl, H., Stoll, B., Valavanoglu, A., and Wiedemann, M.: The THEMIS fluxgate magnetometer, Space Sci. Rev., 141, 235-264, doi:10.1007/s11214-008-9365-9, 2008.

Baker, D. N., Pulkkinen, T. I., Angelopoulos, V., Baumjohann, W., and McPherron, R. L.: Neutral line model of substorms: Past results and present view, J. Geophys. Res., 101, 12975-13010, 1996.

Baumjohann, W. and Glassmeier, K. H.: The transient response mechanism and Pi2 pulsations at substorm onset - Review and outlook, Planet. Space Sci., 32, 1361-1370, 1984.

Caan, M. N., McPherron, R. L., and Russell, C. T.: Substorm and interplanetary magnetic field effects on the geomagnetic tail lobes, J. Geophys. Res., 80(1), 191-194, 1975.

Cheng, C.-C., Chao, J.-K., and Hsu, T.-S.: Evidence of the coupling of a fast magnetospheric cavity mode to field line resonances, Earth Planet. Space, 50, 683-697, 1998.

Cheng, C.-C., Chao, J.-K., and Yumoto, K.: Spectral power of lowlatitude $\mathrm{Pi} 2$ pulsations at $210^{\circ}$ magnetic meridian stations and plasmaspheric cavity resonances, Earth Planet. Space, 52, 615$627,2000$.

Cheng, C.-C., Russell, C. T., Connors, M., and Chi, P. J.: Relationship between multiple substorm onsets and the IMF: A case study, J. Geophys. Res., 107, 1289, doi:10.1029/2001JA007553, 2002a.

Cheng, C.-C., Russell, C. T., Gao, Y. F., and Chi, P. J.: On consecutive bursts of low-latitude Pi2 pulsations, J. Atmos. Sol.-Terr. Phys., 64, 1809-1821, 2002b.

Cheng, C.-C., Russell, C. T., Yumoto, K., Gao, Y. F., and Chi, P. J.: Characteristics of consecutive bursts of $\mathrm{Pi} 2$ pulsations observed at the SMALL array: A new implication, Earth Planet. Space, 56, 531-545, 2004.

Cheng, C.-C., Russell, C. T., Reeves, G. D., Connors, M., and Moldwin, M. B.: On the relationship between doubleonset substorm, pseudobreakup, and IMF variation: The 4 September 1999 event, J. Geophys. Res., 110, A07201, doi:10.1029/2004JA010778, 2005.

Cheng, C.-C., Russell, C. T., Angelopoulos, V., Mann, I., Glassmeier, K. H., Auster, U., and Baumjohann, W.: THEMIS observations of consecutive bursts of $\mathrm{Pi} 2$ pulsations: The
20 April 2007 event, J. Geophys. Res., 114, A00C19, doi:10.1029/2008JA013538, 2009a.

Cheng, C.-C., Russell, C. T., and Shue, J.-H.: On the association of quiet-time Pi2 pulsations with IMF variations, Adv. Space Res., 43, 1118-1129, doi:10.1016/j.asr.2008.12.001, 2009 b.

Clauer, C. R. and McPherron, R. L.: Mapping the local timeuniversal time development of magnetospheric substorms using mid-latitude magnetic observations, J. Geophys. Res., 79, 28112820, 1974.

Glassmeier, K.-H.: Ground-based observations of field-aligned currents in auroral zone - Methods and results, Ann. Geophys., 5, 115-125, 1987.

Glassmeier, K.-H., Baumjohann, W., Korth, A., and Gough, P.: High-latitude Pi2 pulsations, ELF intensity, and particle flux variations - A case study, Ann. Geophys., 6, 287-295, 1988.

Grocott, A., Yeoman, T. K., Milan, S. E., and Cowley, S. W. H.: Interhemispheric observations of the ionospheric signature of tail reconnection during IMF-northward non-substorm intervals, Ann. Geophys., 23, 1763-1770, doi:10.5194/angeo-231763-2005, 2005.

Hones Jr., E. W.: Plasma sheet behavior during substorms, in Magnetic Reconnection in Space and Laboratory Plasmas, Geophys. Monogr. Ser., 30, edited by: Hones Jr., E. W., p. 178, AGU, Washington D.C., 1984.

Hsu, T.-S. and McPherron, R. L.: An evaluation of the statistical significance of the association between northward turnings of the IMF and substorm expansion onsets, J. Geophys. Res., 107, 1398, doi:1310.1029/2000JA000125, 2002.

Itonaga, M. and Yumoto, K.: ULF waves and the ground magnetic field, J. Geophys. Res., 103, 9285-9291, 1998.

Kan, J. R.: A global integrated substorm model: Tail reconnection and magnetoshere-ionosphere coupling, J. Geophys. Res., 103, 11787-11795, 1998.

Kepko, L., Kivelson, M. G., McPherron, R. L., and Spence, H. E.: Relative timing of substorm onset phenomena, J. Geophys. Res., 109, A04203, doi:10.1029/2003JA010285, 2004.

Lester, M., Hughes, W. J., and Singer, H. J.: Polarization patterns of Pi2 magnetic pulsations and the substorm current wedge, J. Geophys. Res., 88, 7958-7966, 1983.

Lester, M., Hughes, W. J., and Singer, H. J.: Longitudinal structure in $\mathrm{Pi} 2$ pulsations and the substorm current wedge, J. Geophys. Res., 89, 5489-5494, 1984.

Lester, M., Singer, H. J., Smits, D. P., and Hughes, W. J.: Pi2 pulsations and the substormcurrent wedge - Low-latitude polarization, J. Geophys. Res., 94, 17133-17141, 1989.

Lin, C. A., Lee, L. C., and Sun, Y. J.: Observations of Pi2 pulsations at a very low latitude $(L=1.06)$ station and magnetospheric cavity resonances, J. Geophys. Res., 96, 21105-21114, 1991.

Lui, A. T. Y.: Electric current approach to magnetospheric physics and the distinction between current disruption and magnetic reconnection, in: Magnetospheric Current Systems, Geophys. Monogr. Ser., 118, edited by: Ohtani, S.-I., Fujii, R., Hesse, M., and Lysak, R. L., p. 31, AGU, Washington D.C., 2000.

Lyons, L. R.: Substorms: fundamental observation features, distinction from other disturbances and external triggering, J. Geophys. Res., 101, 13011-13026, 1996.

Lyons, L. R., Blanchard, G. T., Samson, J. C., Lepping, R. P., Yamaoto, T., and Moretto, T.: Coordinated observations demonstrating external substorm triggering, J. Geophys. Res., 102, 
27039-27051, 1997.

Mann, I. R., Milling, D. K., Rae, I. J., Ozeke, L. G., Kale, A., Kale, Z. C., Murphy, K. R., Parent, A., Usanova, M., Pahud, D. M., Lee, E.-A., Amalraj, V., Wallis, D. D., Angelopoulos, V., Glassmeier, K.-H., Russell, C. T., Auster, H.-U., and Singer, H. J.: The upgraded CARISMA magnetometer array in the THEMIS era, Space Sci. Rev., 141, 413-451, doi:10.1007/s11214-008-94576, 2008.

McPherron, R. L., Terasawa, T., and Nishida, A.: Solar wind triggering of substorm expansion onset, J. Geomag. Geoelectr., 38, 1089-1108, 1986.

Mishin, V. M., Saifudinova, T., Bazarzhapov, A., Russell, C. T., Baumjohann, W., Nakamura, R., and Kubyshkina, M.: Two distinct substorm onsets, J. Geophys. Res., 106, 13105-13118, 2001.

Morley, S. K. and Freeman, M. P.: On the association between northward turnings of the interplanetary magnetic field and substorm onsets, Geophys. Res. Lett., 34, L08104, doi:10.1029/2006GL028891, 2007.

Olson, J. V.: Pi2 pulsations and substorm onsets: A review, J. Geophys. Res., 104, 17499-17520, 1999.

Rostoker, G.: Identifications of substorm expansive phase onsets, J. Geophys. Res., 107, 1137, doi:10.1029/2001JA003504, 2002.

Russell, C. T.: The solar wind and magnetospheric dynamics, in: Correlated Interplanetary and Magnetospheric Observation, edited by: Page, D. E., p. 3, D Reidel, Holland, 1974.

Russell, C. T.: How northward turnings of the IMF can lead to substorm expansion onsets, Geophys. Res. Lett., 27, 3257-3259, 2000.

Russell, C. T. and McPherron, R. L.: The magnetotail and substorms, Space Sci. Rev., 15, 205-266, 1973.

Russell, C. T., Wang, Y. L., and Raeder, J.: Possible dipole tilt dependence of dayside magnetopause reconnection, Geophys. Res. Lett., 30, 1937-1940, doi:10.1029/2003GL017725, 2003.
Russell, C. T., Chi, P. J., Dearborn, D. J., Ge, Y. S., Kuo-Tiong, B., Means, J. D., Pierce, D. R., Rowe, K. M., and Snare, R. C.: THEMIS ground-based magentometers, Space Sci. Rev., 141, 389-412, doi:10.1007/s11214-008-9337-0, 2008.

Saito, T.: Geomagnetic pulsations, Space Sci. Rev., 10, 319-412, 1969.

Sakurai, T. and McPherron, R. L.: Satellite observations of Pi2 activity at synchronous orbit, J. Geophys. Res., 88, 7015-7024, 1983.

Sergeev, V. A., Dmitrieva, N. P., and Barkova, E. S.: Triggering of substorm expansion by the IMF directional discontinuities: time delay analysis, Planet. Space Sci., 34, 1109-1118, 1986.

Shiokawa, K., Baumjohann, W., Haerendel, G., Paschmann, G., Fennell, J. F., Friis-Christensen, E., Lühr, H., Reeves, G. D., Russell, C. T., Sutcliffe, P. R., and Takahashi, K.: High-speed flow, substorm current wedge, and multiple Pi2 pulsations, J. Geophys. Res., 103, 4491-4507, 1998.

Takahashi, K., Anderson, R. R., and Hughes, W. J.: Pi2 pulsations with second harmonic: CRRES observations in the plasmasphere, J. Geophys. Res., 108, 1242, doi:10.1029/2003JA009847, 2003.

Untiedt, J. and Baumjohann, W.: Studies of polar current systems using the IMS scandinavian magnetometer array, Space Sci. Rev., 63, 245-390, 1993.

Weimer, D. R., Ober, D. M., Maynard, N. C., Collier, M. R., McComas, D. J., Ness, N. F., Smith, C. W., and Watermann, J.: Predicting interplanetary magnetic field (IMF) propagation delay times using the minimum variance technique, J. Geophys. Res., 108, 1026, doi:10.1029/2002JA009405, 2003.

Yumoto, K.: Generation and propagation mechanisms of lowlatitude magnetic pulsations - A review, J. Geophys., 60, 79-105, 1986. 